Calabazas Creek Research, Inc.

690 Port Drive

San Mateo, CA 94404

\title{
DE-SC0001970
}

\section{Improved Space Charge Modeling for Simulation and Design of Photoinjectors}

\author{
Topic Number: 38 \\ Subtopic Number: g \\ Principal Investigator: Dr. Robert H. Jackson
}

19 April 2010 


\section{Summary of Phase I Findings}

The Local Taylor Polynomial (LTP) technique was explored and tested during Phase I of this program. LTP offers a number of improvements and unique features compared to conventional finite difference. Here, an outline of key results is given. Details are presented below.

\section{- Powerful, systematic solution weight generation techniques}

o A general matrix formulation for computing solution weights was developed

o Optimum field solution weights depend only on the PDE and grid geometry

o Space charge effects included on an equal footing with the field

- Systematic formulation of high order interpolation and electric field computation

o General matrix formulation for field interpolation

o General matrix formulation for electric field computation

o Uniform cylindrical grid expressions illuminate space charge effects

- Better handling of space charge by LTP:

o Consistent methodology for addressing a variety of source issues

o Better error convergence versus grid size (Gaussian beam profile)

o At least $5 \times$ lower maximum error (Gaussian beam profile)

o Analytic treatment of charge using discrete polynomial-shaped particles

- Flexibility in application of advanced grid techniques:

o LTP not tied to any particular grid structure

o Can handle non-conformal boundaries

o Can handle square, rectangular and non-uniform orthogonal grids

o Compatible with multi-level subgrids, grid patches, and adaptive grids

- Applicable to other physics PDEs

o Reduced Helmholtz PDE (eigenvalue)

o Time dependent Helmholtz PDE

o Maxwell Equations 


\section{Introduction}

Photoinjectors play a key role in high-energy accelerators in reducing beam energy spread, increasing beam intensity and enhancing the photon flux from undulators. There are substantial differences in time and spatial scales between the overall photoinjector accelerating structure and the photo-emitted electron bunch making detailed time-dependent simulations of bunch formation and propagation difficult. In fact, simulations of bunch evolution are often performed separately from simulations of the accelerating structure [1]. With sub-millimeter/picosecond bunches and tens-of-centimeter/GHz RF cavities the space/time scales are disparate enough to make simulations difficult but close enough that the long-time scale cannot be treated as DC, especially because of the low electron velocities during the initial bunch formation and acceleration process. Additionally, at the photocathode the feedback of space charge forces from the bunch front strongly influences the photo-injection process. Hence, simultaneous computation of effects at both scales is needed.

Because of the (mostly) cylindrical symmetry of photoinjector components and the charge bunch the use of cylindrical coordinates can provide significant benefits. Specifically, 2D cylindrical simulation can be an extremely efficient design tool permitting high space/time resolution while incorporating almost all of the relevant physics. Appropriately combined with 3D simulation (in cylindrical and/or rectangular coordinates) the resulting simulation capability offers both efficiency and fidelity for exploring concepts as well as pursuing specific designs. This is contingent upon the accurate representation of space charge and electric fields near the axis in cylindrical coordinates (both 2D and 3D) where the electron bunch is located.

It has long been known that the curvature of $(r, z)$ and $(r, \varphi, z)$ coordinates, Fig. 1, requires adjustment of solution coefficients and particle/current weights as functions of distance from the axis. Recent progress on particle interpolation [2] and field solution [3] in cylindrical coordinates has provided a consistent methodology for development of accurate numerical techniques. Interpolating particle charges and currents to mesh nodes as the source terms to Maxwell's equations in a particle-in-cell (PIC) code using the standard scheme results in systematic errors for curvilinear coordinates. Likewise, improved field solutions, obtained by a Taylor polynomial approach [3], show that additional, radius-dependent corrections are necessary in axisymmetric coordinates.

Improved space charge algorithms alone are insufficient to resolve the electron bunch formation and evolution to the desired degree. High-resolution grids are necessary to deal with subpicosecond laser pulses and sub-millimeter bunch lengths. However, using such fine grid resolution throughout a simulation needlessly increases the runtime and required computational resources. The use of subgrids to provide high resolution only where needed is well established in many fields but has not seen widespread use in the PIC community. The reasons for this vary but among them is the complexity of accurately dealing with particles entering and exiting regions of different resolution. In addition, for fully time dependent simulations there are fundamental field stability issues with subgrids that must be handled before particles are even introduced into the problem. The effect of particles on stability is a further concern. 


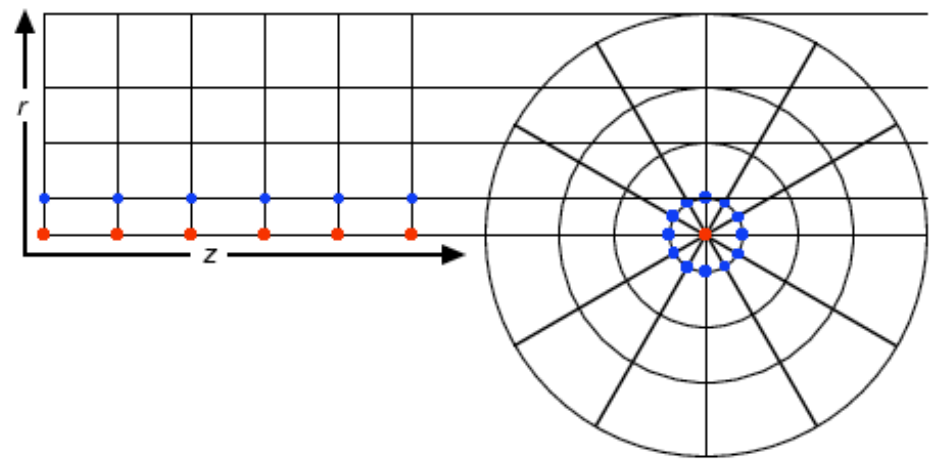

Figure 1: Curvature and special grid nodes in cylindrical coordinates. Note that there are three distinct node types: 1) axis nodes (red), 2) first-row nodes (blue) and 3) "standard" nodes. To obtain the highest solution accuracies the axis and first row nodes require separate treatment.

\section{The Local Taylor Polynomial Method}

The Local Taylor Polynomial (LTP) method is similar to standard finite differences in that both can be related to a Taylor expansion of the solution function around a point. One key difference is that LTP is based on a local analytic solution (polynomial in this initial work) of the continuum partial differential equation (PDE). Therefore, the polynomial satisfies the PDE at each order but is not necessarily an exact solution because it has to be truncated at some finite order. Discrete numerical formulas are then developed using this analytic solution polynomial. In contrast, finite difference formulas enforce the "solution" through discrete approximation of the PDE. One key benefit of using a polynomial rather than other local representations is the simplicity of taking derivatives. However, other representations may require fewer terms to achieve equivalent fidelity, to be explored in future work.

An important benefit of the LTP approach is that the source function becomes a full component in the formulation, on an equal footing with the field function. This provides a consistent methodology for addressing source effects in a variety of situations, e.g. near symmetry boundaries such as the cylindrical axis, or near PEC or dielectric boundaries. This capability is lacking in standard finite difference developments.

We begin our exposition of the LTP technique using the 1-D scalar Poisson PDE in cylindrical coordinates. This 1-D example serves to highlight features of the LTP method without the distracting details of higher dimensions. Also, we will restrict the discussion to the Poisson PDE, but note that the method is quite general and applicable to other PDE's.

Following this introduction, a general matrix methodology for implementing the LTP method will be developed and discussed. Examples will be given for 2-D cylindrical coordinates and some special cases will be addressed, e.g. on-axis and first-row formulas, non-conformal boundaries, etc. Application of the LTP to electric field calculation is then examined.

\subsection{The Local Taylor Polynomial}

LTP starts with general polynomials for the field and source functions written in terms of "offset" coordinates (the "local" in LTP) as shown in the expressions below. Here $r_{P}$ is the 
reference, or primary, node, $\eta$ is the normalized offset from the primary node, $h$ is a (somewhat arbitrary) scale factor ${ }^{1}$ and $\delta$ is the cylindrical curvature factor.

$$
\begin{array}{rlrl}
\eta & =\left(r-r_{p}\right) / h & \delta & =h / r_{p} \\
U(\eta) & =\sum_{i=0}^{\infty} a_{i} \eta^{i} & S(\eta) & =\sum_{i=0}^{\infty} b_{i} \eta^{i}
\end{array}
$$

It is clear that these polynomials may or may not be the best representations of the potential and source functions in the "global" sense. What we are after is a representation over a local, homogeneous region that is efficient for use with the PDE.

The next step is substitution of these polynomials into the localized PDE and collection of terms with like powers of the offset variable. The localized 1-D Poisson PDE away from the axis in cylindrical coordinates is shown below.

$$
\frac{\partial^{2} U}{\partial \eta^{2}}+\delta \eta \frac{\partial^{2} U}{\partial \eta^{2}}+\delta \frac{\partial U}{\partial \eta}=-S-\delta \eta S
$$

Polynomial substitution and the requirement that the PDE be satisfied exactly at all points in the region surrounding the primary node results in the general coefficient relationship below, where $0 \leq i<\infty$ and $b_{-1} \equiv 0$.

$$
a_{i+2}=-\frac{1}{(i+2)(i+1)} b_{i}-\frac{1}{(i+2)(i+1)} \delta b_{i-1}-\frac{i+1}{i+2} \delta a_{i+1}
$$

Back substituting this relation into the potential produces a polynomial with series multipliers of the original coefficients of the following form:

$$
C_{n}=1-\frac{1}{n+1} \delta \eta+\frac{1}{n+2} \delta^{2} \eta^{2}-\frac{1}{n+3} \delta^{3} \eta^{3}+\ldots
$$

Extending the polynomial to infinite order, and assuming these series relations maintain the same form, we arrive at the relations shown below for the local polynomial that exactly satisfies the continuum PDE at the primary node. It is clear that a one-to-one correspondence can be drawn between this polynomial and the Taylor series for $U$ at the primary node, hence the terminology Local Taylor Polynomial. Although this example is 1-D, many features of the coefficients carry over to 2-D with only minor changes. Also, note that only the first two terms include coefficients from the potential polynomial, all the other terms come from the space charge polynomial.

$$
\begin{gathered}
U(\eta)=U_{P}+C_{1}(\delta \eta) a_{1} \eta-\eta^{2} \sum_{i=0}^{\infty} \frac{C_{(i+2)}(\delta \eta)}{(i+2)(i+1)} \eta^{i} b_{i} \\
C_{i}(z)=1+\sum_{n=1}^{\infty}(-1)^{n} \frac{z^{n}}{n+i}
\end{gathered}
$$

Numerical formulas can now be developed by evaluating the polynomial at sets of discrete points (stencils). This can be done by "hand", which we will demonstrate first, or using matrix

${ }^{1}$ For uniform square grids, $h$ is always the grid spacing, but it is really more general than this as will be shown in the discussion on non-uniform grids. 
algebra, which will be developed below. For uniform grids in 1-D, the north and south points are at $\eta= \pm 1$ respectively and the potential values at the stencil points are related by:

$$
\begin{aligned}
& U_{N}=U_{P}+C_{1}(\delta) a_{1}-\sum_{i=0}^{\infty} \frac{C_{(i+2)}(\delta)}{(i+2)(i+1)} b_{i} \\
& U_{P}=U_{P} \\
& U_{S}=U_{P}-C_{1}(-\delta) a_{1}-\sum_{i=0}^{\infty} \frac{C_{(i+2)}(-\delta)}{(i+2)(i+1)}(-1)^{i} b_{i}
\end{aligned}
$$

These relations can be used to estimate a value for the potential at the primary node based on values at the north and south points, as shown in the expression below.

$$
\begin{aligned}
U_{P}= & \frac{C_{1}(-\delta) U_{N}+C_{1}(\delta) U_{S}+\frac{1}{2}\left[C_{1}(-\delta) C_{2}(\delta)+C_{1}(\delta) C_{2}(-\delta)\right] b_{0}}{\left[C_{1}(-\delta)+C_{1}(\delta)\right]} \\
& +\frac{\left[C_{1}(-\delta) C_{3}(\delta)-C_{1}(\delta) C_{3}(-\delta)\right]}{6\left[C_{1}(-\delta)+C_{1}(\delta)\right]} b_{1} \\
& +\sum_{i=2}^{\infty} \frac{\left[C_{1}(-\delta) C_{(i+2)}(\delta)+(-1)^{i} C_{1}(\delta) C_{(i+2)}(-\delta)\right]}{(i+2)(i+1)\left[C_{1}(-\delta)+C_{1}(\delta)\right]} b_{i}
\end{aligned}
$$

Given the two potential $\left(U_{N}, U_{S}\right)$ and three source $\left(S_{N}, S_{P}, S_{S}\right)$ values available at the stencil points, only the $b_{0}$ (i.e. $S_{P}$ ) and $b_{1}$ (i.e. $\left[S_{N}-S_{S}\right] / 2$ ) source polynomial terms can be computed. The infinite sum is dropped (i.e. truncated at $i=2$ ) and the discrete version of (0.9) becomes:

$$
\begin{aligned}
U_{P}= & \frac{C_{1}(-\delta) U_{N}+C_{1}(\delta) U_{S}+\frac{1}{2}\left[C_{1}(-\delta) C_{2}(\delta)+C_{1}(\delta) C_{2}(-\delta)\right] S_{P}}{\left[C_{1}(-\delta)+C_{1}(\delta)\right]} \\
& +\frac{\left[C_{1}(-\delta) C_{3}(\delta)-C_{1}(\delta) C_{3}(-\delta)\right]}{6\left[C_{1}(-\delta)+C_{1}(\delta)\right]} \frac{S_{N}-S_{S}}{2}+H O T S
\end{aligned}
$$

The coefficients in this expression look complex; however, they are constants that depend only on the number of grid cells away from the axis, i.e. $\delta$. In 2-D/3-D cases, these coefficients only need to be computed once for each radius and are valid for all nodes at that radius.

Already we see at least two differences between this formula and the standard finite difference form: 1) the complex coefficients for the potential values and primary node space charge, and 2) the presence of the $b_{1}$ space charge term to achieve full $2^{\text {nd }}$ order. The first indicates that LTP takes into account the "hidden" curvature of $(r, z)$ cylindrical coordinates and the second shows that space charge is treated in a manner consistent with both the geometry and the governing PDE.

\subsection{Analytic Coefficient Terms}

It is worthwhile to reexamine the $C_{i}$ series defined above. We note that the infinite sum in these terms is similar to a natural log series as shown below. 


$$
\ln (1+z)=-\sum_{n=1}^{\infty}(-1)^{n} \frac{z^{n}}{n}
$$

Using this expression, the infinite sum in the coefficient terms can be replaced with the natural $\log$ function and a finite sum.

$$
C_{i}(z)=1-(-1)^{i} z^{-i}\left[\ln (1+z)+\sum_{n=1}^{i}(-1)^{n} \frac{z^{n}}{n}\right]
$$

Either form can be used depending on evaluation factors. Near the axis, where " $z$ " would be large, the analytic form should be more accurate. Far from the axis, where " $z$ " is small, the infinite sum should converge rapidly and the original form might be best. Note that a problem occurs in the analytic expression if $z=-1$, which is addressed in the section below on special cases.

One final observation concerning the analytic terms, specifically the LTP term involving $C_{1}(z)$. This expression is shown below after some manipulations that clearly show the connection of this term to a coaxial $\log (\mathrm{r})$ form. In other words, away from the axis, LTP models a local coaxial potential distribution around the primary node modified by local space charge.

$$
\eta C_{1}(\delta \eta) a_{1}=\frac{1}{\delta} \ln (1+\delta \eta) a_{1}=\frac{r_{P}}{h} \ln \left(\frac{r}{r_{p}}\right) a_{1}
$$

\subsection{Local Taylor Polynomial in 2-D Cylindrical Coordinates}

In cylindrical $(r, z)$ coordinates the localized PDE and polynomials become:

$$
\begin{gathered}
{\left[\frac{\partial^{2} U}{\partial \varepsilon^{2}}+\frac{\partial^{2} U}{\partial \eta^{2}}+S\right]+\delta \frac{\partial U}{\partial \eta}+\delta \eta\left[\frac{\partial^{2} U}{\partial \varepsilon^{2}}+\frac{\partial^{2} U}{\partial \eta^{2}}+S\right]=0} \\
U=\sum_{i=0}^{M} \sum_{j=0}^{i} a_{i j} \varepsilon^{(i-j)} \eta^{j} \quad S=\sum_{i=0}^{N} \sum_{j=0}^{i} b_{i j} \varepsilon^{(i-j)} \eta^{j}
\end{gathered}
$$

Applying the LTP method to these equations results in the following 2-D cylindrical LTP for the scalar Poisson PDE.

$$
\begin{aligned}
U(\varepsilon, \eta) & =U_{P}+a_{10} \varepsilon+\eta C_{1}(\delta \eta) a_{11}+\left(\varepsilon^{2}-\eta^{2} C_{2}(\delta \eta)\right) a_{20} \\
& -\frac{1}{2} \eta^{2} C_{2}(\delta \eta) S_{P}-\frac{1}{2} \varepsilon \eta^{2} C_{2}(\delta \eta) b_{10}-\frac{1}{6} \eta^{3} C_{3}(\delta \eta) b_{11} \\
& +\varepsilon \eta C_{1}(\delta \eta) a_{21}+\varepsilon\left(\varepsilon^{2}-3 \eta^{2} C_{2}(\delta \eta)\right) a_{30} \\
& +\eta\left[\varepsilon^{2}\left(1+\frac{1}{3} \delta^{2} \eta^{2}\right)-\frac{1}{3} \eta^{2}\left(1+\frac{7}{20} \delta^{2} \eta^{2}\right)\right] a_{31} \\
& -\frac{1}{2} \delta \eta^{2}\left[\varepsilon^{2}\left(1+\frac{1}{2} \delta^{2} \eta^{2}\right)-\frac{1}{3} \eta^{2}\left(1+\frac{11}{20} \delta^{2} \eta^{2}\right)\right] a_{31}
\end{aligned}
$$

In order to simplify derivations and formulas, the terms in this expression will be written as follows when evaluated at the north and south points of a square stencil. The east and west stencil points are straightforward.

$$
U_{N}=U_{P}+k_{1} a_{11}-k_{2} a_{20}-\frac{1}{2} k_{2} S_{P}-\frac{1}{6} m_{1} b_{11}-k_{3} a_{31}
$$




$$
\begin{gathered}
U_{S}=U_{P}-g_{1} a_{11}-g_{2} a_{20}-\frac{1}{2} g_{2} S_{P}+\frac{1}{6} n_{1} b_{11}+g_{3} a_{31} \\
U_{E}=U_{P}+a_{10}+a_{20}+a_{30} \\
U_{W}=U_{P}-a_{10}+a_{20}-a_{30}
\end{gathered}
$$

The modified coefficient terms are shown below.

$$
\begin{aligned}
k_{1} & =C_{1}(\delta) & g_{1} & =C_{1}(-\delta) \\
k_{2} & =C_{2}(\delta) & g_{2} & =C_{2}(-\delta) \\
k_{3} & =\frac{1}{3}\left(1-\frac{1}{2} \delta+\frac{7}{20} \delta^{2}-\frac{11}{40} \delta^{3}\right) & g_{3} & =\frac{1}{3}\left(1+\frac{1}{2} \delta+\frac{7}{20} \delta^{2}+\frac{11}{40} \delta^{3}\right) \\
m_{1} & =C_{3}(\delta) & n_{1} & =C_{3}(-\delta)
\end{aligned}
$$

The $2^{\text {nd }}$ order solution formula for a square stencil using this notation is:

$$
\begin{gathered}
U_{p}=\frac{C_{N} U_{N}+C_{s} U_{S}+C_{E W} S_{p}+C_{E W}\left(U_{E}+U_{W}\right)}{C_{D}} \\
C_{N}=g_{1} \quad C_{S}=k_{1} \quad C_{E W}=\frac{1}{2}\left(g_{1} k_{2}+g_{2} k_{1}\right) \\
C_{D}=\left(g_{1} k_{2}+g_{2} k_{1}\right)+\left(k_{1}+g_{1}\right)
\end{gathered}
$$

These solution coefficients will be referred to in the examples given below.

\subsection{Matrix Computation of Solution Coefficients}

The developments presented above illustrate how the LTP can be used to derive discrete formulas for accurate computation of potentials and fields. Direct manipulation of the polynomials in the above manner is fine for low-order uniform stencils. However, in more general cases, such as higher-order and non-conformal stencils, more systematic methods for deriving formula coefficients are needed. It this section, a formal solution method will be developed based on matrix algebra. This development will use the 2-D polynomials but applies to both 1-D and 3-D with only minor adjustments.

The processes for determining solution and interpolation coefficients can be cast as matrix problems in the following manner. First, generate an indexing scheme for the polynomial coefficients from a two-index to a one-index scheme (implementation details are unimportant). The field and source offset terms multiplying each coefficient are written as indexed functions of the evaluation point and the coefficient as shown below. Note that the field-derived and sourcederived coefficients have been separated.

$$
U_{i}=U\left(\varepsilon_{i}, \eta_{i}, \delta_{i}\right)=\sum_{j=0}^{M} \alpha_{i j}\left(\varepsilon_{i}, \eta_{i}, \delta_{i}\right) A_{j}-\sum_{j=0}^{N} \beta_{i j}\left(\varepsilon_{i}, \eta_{i}, \delta_{i}\right) B_{j}
$$

This equation is rearranged to move space-charge effects (addressed below) to the left-hand side of the equation. In what follows, keep in mind that at the primary node, i.e. $(\varepsilon, \eta)=(0,0)$, the potential satisfies $U_{p}=\phi_{p}=A_{0}=a_{00}$.

$$
\phi_{i}=U_{i}+\sum_{j=0}^{N} \beta_{i j} B_{j}=\sum_{j=0}^{M} \alpha_{i j} A_{j}
$$


This relation can be written as a matrix equation as shown below where $\boldsymbol{\Phi}$ is a vector of (spacecharge adjusted) potential values at the stencil points, $\mathbf{A}$ is a vector of the LTP potential coefficients and $\mathbf{X}$ is a matrix of LTP offset terms for the potential.

$$
\Phi=\mathbf{X A}
$$

The components of this equation are detailed below where $L$ is the number of points in the stencil and $M$ is the number of unknown polynomial coefficients. Note should be taken that the integers $L$ and $M$ are not necessarily equal and, hence, the offset matrix $\mathbf{X}$ may not be square. (X can be made square if either stencil points or polynomial coefficients are ignored.)

$$
\begin{gathered}
\Phi^{\mathbf{T}}=\left(\phi_{0}, \phi_{1}, \cdots, \phi_{L}\right) \\
\mathbf{A}^{\mathrm{T}}=\left(A_{0}, A_{1}, \cdots, A_{M}\right) \\
\mathbf{X}=\left(\begin{array}{cccc}
\alpha_{10} & \alpha_{11} & \cdots & \alpha_{1 M} \\
\alpha_{20} & \alpha_{21} & \cdots & \alpha_{2 M} \\
\vdots & \vdots & \ddots & \vdots \\
\alpha_{L 0} & \alpha_{L 1} & \cdots & \alpha_{L M}
\end{array}\right)
\end{gathered}
$$

Solving for the primary node potential amounts to multiplying the surrounding potentials by weights and summing. The issue is determining the optimum weights for a given stencil. The matrix relation for this can be stated as follows:

$$
\phi_{p}=\mathbf{w}^{\mathrm{T}} \Phi=\mathbf{w}^{\mathrm{T}} \mathbf{X A}
$$

Note that in the coefficient vector $\mathbf{A}$ the first coefficient is the primary node potential, i.e. $A_{0}=\phi_{p}$, hence, what is needed is for the product of the weight vector and the offset matrix to yield a vector that has all elements equal to zero (or nearly so) except for the first element, which is one. Expressed in matrix form, this requirement is shown below where $\mathbf{r}$ is the "residual" vector.

$$
\mathbf{w}^{\mathbf{T}} \mathbf{X}=\mathbf{r}^{\mathbf{T}}=(1,0, \cdots, 0)
$$

The optimum weights are determined by solving this matrix relation. The weighting process done in this manner is equivalent to "zeroing" higher order terms in the Taylor expansion of the potential function. Taking the transpose of both sides of the weight relation produces the expression below.

$$
\mathbf{X}^{\mathrm{T}} \mathbf{W}=\mathbf{r}
$$

If $\mathbf{X}$ is square, the weight solution is the first column of the inverse of $\mathbf{X}$-transpose.

$$
\mathbf{w}=\left(\mathbf{X}^{\mathbf{T}}\right)^{-1} \mathbf{r}
$$

If $\mathbf{X}$ is non-square a different approach is required. Express $\mathbf{w}$ as the product of $\mathbf{X}$ and an unknown vector $\mathbf{v}$, i.e. $\mathbf{w}=\mathbf{X v}$. This produces the square matrix $\mathbf{X}^{\mathbf{T}} \mathbf{X}$, which can be inverted. A solution for $\mathbf{v}$ is obtained and substituted back into the expression for $\mathbf{w}$, yielding the solution 
shown below. This is called the generalized matrix inverse and is valid if the number of stencil points exceeds the number of coefficients.

$$
\mathbf{w}=\left[\mathbf{X}\left(\mathbf{X}^{\mathrm{T}} \mathbf{X}\right)^{-1}\right] \mathbf{r}
$$

The key feature here is that this expression does not depend on the potential or space charge in any way. Thus, the solution weights depend only on the PDE and the geometric layout of the stencil relative to the primary node. As a consequence, the weights can be calculated only once for a given configuration.

Weight quality can be tested by computing the product of $\mathbf{X}^{\mathbf{T}}$ and the weight vector. The difference between this product and the ideal residual vector provides a measure of weight quality. For accurate weights, the differences should have magnitudes on the order of the roundoff precision for the software implementation, e.g. about $10^{-14}$ or less for double precision.

$$
\varepsilon=\mathbf{r}-\mathbf{X}^{\mathrm{T}} \mathbf{w}
$$

Although the weights themselves are not affected by space charge, the potential calculation is. The primary node potential is obtained from the relation below where $\mathbf{Q}$ is the portion of the LTP offset matrix due to the source and $\mathbf{B}$ the source coefficient vector.

$$
U_{p}=\mathbf{w}^{\mathbf{T}} \mathbf{U}+\mathbf{w}^{\mathbf{T}} \mathbf{Q B}
$$

Obviously there are constraints on the offset matrices to ensure valid and accurate results. In particular, for symmetric stencils certain terms in the offset matrix will be zero for all points in the stencil. As an example, in the classic 5-point stencil all "cross" terms, i.e. $\varepsilon^{i} \eta^{j}$ with $i$ and $j \neq$ 0 , are zero at the stencil points. Thus, the offset matrix should be constructed to eliminate allzero columns and their associated polynomial coefficients.

Shown below are examples of the elements in the coefficient vectors and offset matrices for the potential (A and $\mathbf{X})$ and source (B and $\mathbf{Q})$.

$$
\mathbf{A}^{\mathbf{T}}=\left(a_{00}, a_{10}, a_{11}, a_{20}, \cdots, a_{41}\right)
$$

$$
\begin{aligned}
\alpha_{i 0}= & 1 \\
\alpha_{i 1}= & \varepsilon \\
\alpha_{i 2} & =\eta\left(1-\frac{1}{2} \delta \eta+\frac{1}{3} \delta^{2} \eta^{2}-\frac{1}{4} \delta^{3} \eta^{3}+\frac{1}{5} \delta^{4} \eta^{4}-\frac{1}{6} \delta^{5} \eta^{5}\right) \\
\alpha_{i 3} & =\left(\varepsilon^{2}-\eta^{2}\left[1-\frac{1}{3} \delta \eta+\frac{1}{4} \delta^{2} \eta^{2}-\frac{1}{5} \delta^{3} \eta^{3}+\frac{1}{6} \delta^{4} \eta^{4}\right]\right) \\
\alpha_{i 4}= & \varepsilon \eta\left(1-\frac{1}{2} \delta \eta+\frac{1}{3} \delta^{2} \eta^{2}-\frac{1}{4} \delta^{3} \eta^{3}+\frac{1}{5} \delta^{4} \eta^{4}\right) \\
\alpha_{i 5}= & \varepsilon\left(\varepsilon^{2}-3 \eta^{2}\left[1-\frac{1}{3} \delta \eta+\frac{1}{4} \delta^{2} \eta^{2}-\frac{1}{5} \delta^{3} \eta^{3}\right]\right) \\
\alpha_{i 6}= & \left(\eta\left[\varepsilon^{2}-\frac{1}{3} \eta^{2}+\frac{1}{3} \delta^{2} \varepsilon^{2} \eta^{2}-\frac{7}{60} \delta^{2} \eta^{4}\right]\right. \\
& \left.-\frac{1}{2} \delta \eta^{2}\left[\varepsilon^{2}-\frac{1}{3} \eta^{2}+\frac{1}{2} \delta^{2} \varepsilon^{2} \eta^{2}-\frac{11}{60} \delta^{2} \eta^{4}\right]\right) \\
\alpha_{i 7}= & \left(\varepsilon^{4}-6 \varepsilon^{2} \eta^{2}+\eta^{4}+2 \delta \varepsilon^{2} \eta^{3}-\frac{2}{5} \delta \eta^{5}-\frac{3}{2} \delta^{2} \varepsilon^{2} \eta^{4}+\frac{3}{10} \delta^{2} \eta^{6}\right) \\
\alpha_{i 8} & =\varepsilon \eta\left(\varepsilon^{2}\left[1-\frac{1}{2} \delta \eta+\frac{1}{3} \delta^{2} \eta^{2}\right]-\eta^{2}\left[1-\frac{1}{2} \delta \eta+\frac{7}{20} \delta^{2} \eta^{2}\right]\right)
\end{aligned}
$$




$$
\begin{gathered}
\mathbf{B}^{\mathbf{T}}=\left(b_{00}, b_{10}, b_{11}, b_{20}, b_{21}, b_{22}\right) \\
\beta_{i 0}=\frac{1}{2} \eta^{2}\left(1-\frac{1}{3} \delta \eta+\frac{1}{4} \delta^{2} \eta^{2}-\frac{1}{5} \delta^{3} \eta^{3}+\frac{1}{6} \delta^{4} \eta^{4}\right) \\
\beta_{i 1}=\frac{1}{2} \varepsilon \eta^{2}\left(1-\frac{1}{3} \delta \eta+\frac{1}{4} \delta^{2} \eta^{2}-\frac{1}{5} \delta^{3} \eta^{3}\right) \\
\beta_{i 2}=\frac{1}{6} \eta^{3}\left(1-\frac{1}{4} \delta \eta+\frac{1}{5} \delta^{2} \eta^{2}-\frac{1}{6} \delta^{3} \eta^{3}\right) \\
\beta_{i 3}=\frac{1}{2} \eta^{2}\left(\varepsilon^{2}\left[1-\frac{1}{3} \delta \eta+\frac{1}{4} \delta^{2} \eta^{2}\right]-\eta^{2}\left[\frac{1}{6}-\frac{1}{15} \delta \eta+\frac{1}{20} \delta^{2} \eta^{2}\right]\right) \\
\beta_{i 4}=\frac{1}{6} \varepsilon \eta^{3}\left(1-\frac{1}{4} \delta \eta+\frac{1}{5} \delta^{2} \eta^{2}\right) \\
\beta_{i 5}=\frac{1}{12} \eta^{4}\left(1-\frac{1}{5} \delta \eta+\frac{1}{6} \delta^{2} \eta^{2}\right)
\end{gathered}
$$

Note, the source polynomial coefficients at any given order in the LTP always occur at two orders lower than the field coefficients.

\subsection{Matrix Computation of Space Charge Effects}

Solving for the potential at the primary node requires computing the source terms in the $\mathbf{w}^{\mathbf{T}} \mathbf{Q B}$ portion of the solution equation. The weights are known and the LTP source offset-matrix $\mathbf{Q}$ is easily computed (the $\beta^{\prime}$ s above). It is the source polynomial coefficient vector $\mathbf{B}$ that remains to be determined. $\mathbf{B}$ can be computed using the matrix expression below and the source values at the stencil points.

$$
\mathbf{S}=\mathbf{Z B}
$$

In this expression, $\mathbf{S}$ is the vector of source values at the stencil points, $\mathbf{B}$ is the vector of source polynomial coefficients and $\mathbf{Z}$ is the source polynomial offset matrix, not to be confused with the LTP $\mathbf{Q}$ matrix. The elements for $\mathbf{S}, \mathbf{Z}$ and $\mathbf{B}$ are shown expanded below.

$$
\left(\begin{array}{c}
S_{0} \\
S_{1} \\
\vdots \\
S_{L}
\end{array}\right)=\left(\begin{array}{cccccc}
1 & 0 & 0 & 0 & \mathrm{~K} & 0 \\
1 & \varepsilon_{1} & \eta_{1} & \varepsilon_{1}^{2} & \mathrm{~K} & \varepsilon_{1}^{i} \eta_{1}^{j} \\
\mathrm{M} & \mathrm{M} & \mathrm{M} & \mathrm{M} & \mathrm{O} & \mathrm{M} \\
1 & \varepsilon_{L} & \eta_{L} & \varepsilon_{L}^{2} & \mathrm{~K} & \varepsilon_{L}^{i} \eta_{L}^{j}
\end{array}\right)\left(\begin{array}{c}
b_{00} \\
b_{10} \\
b_{11} \\
b_{20} \\
\mathrm{M} \\
b_{(i+j) j}
\end{array}\right)
$$

Accounting for the possibility that $\mathbf{Z}$ is non-square, a solution can be obtained using the generalized inverse.

$$
\mathbf{B}=\left(\mathbf{Z}^{\mathrm{T}} \mathbf{Z}\right)^{-1} \mathbf{Z}^{\mathrm{T}} \mathbf{S}
$$

The number of source coefficients in $\mathbf{B}$ is determined by the accuracy order but may be larger. This sets a minimum number for the source points but more can be used. Therefore, the potential and space-charge stencils do not have to be the same and the space-charge stencil can include the primary node. Also, because there is no separate relation that reduces the number of independent source coefficients, the total number of source coefficients required to achieve a specified order increases rapidly. 
Substituting this relation into the solution equation yields the expression below where the righthand-side weight vectors, $\mathbf{w}$ and $\boldsymbol{\omega}$, are static, can be pre-computed and only need be computed once at each radius.

$$
\begin{gathered}
U_{p}=\mathbf{w}^{\mathbf{T}} \mathbf{U}+\omega^{\mathbf{T}} \mathbf{S} \\
\omega^{\mathbf{T}}=\mathbf{w}^{\mathbf{T}}\left[\mathbf{Q}\left(\mathbf{Z}^{\mathbf{T}} \mathbf{Z}\right)^{-1} \mathbf{Z}^{\mathbf{T}}\right]
\end{gathered}
$$

The option exists to treat space charge at a different accuracy level than the field, although, in general, the best results will likely be obtained when the field and charge errors are "balanced".

\subsection{Examples ${ }^{2}$}

The matrix technique outlined above will be demonstrated for the square 5-point stencil, i.e. $N=$ $(0,1), S=(0,-1), E=(1,0), W=(-1,0)$ and $P=(0,0)$. The potential and source-density values and polynomials at the $N, S, E$ and $W$ stencil points will be used to construct the vectors and matrices. There are only four points in addition to the central node so a maximum of four coefficients and offset terms can be addressed. Starting with the potential-related terms, the coefficient vector is shown below.

$$
\mathbf{A}^{\mathbf{T}}=\left(a_{00}, a_{10}, a_{11}, a_{20}\right)
$$

Taking the points in $N, S, E, W$ order, the field offset matrix and its transpose are:

$$
\mathbf{X}=\left(\begin{array}{cccc}
1 & 0 & k_{1} & -k_{2} \\
1 & 0 & -g_{1} & -g_{2} \\
1 & 1 & 0 & 1 \\
1 & -1 & 0 & 1
\end{array}\right) \quad \mathbf{X}^{\mathbf{T}}=\left(\begin{array}{cccc}
1 & 1 & 1 & 1 \\
0 & 0 & 1 & -1 \\
k_{1} & -g_{1} & 0 & 0 \\
-k_{2} & -g_{2} & 1 & 1
\end{array}\right)
$$

Note that use has been made of the notation for the offset terms defined in Section 2.3 above. With the aid of mathematical software, the inverse of this square offset-matrix transpose can be determined analytically.

$$
\begin{aligned}
\left(\mathbf{X}^{\mathbf{T}}\right)^{-1} & =\frac{1}{2\left[\left(k_{1} g_{2}+k_{2} g_{1}\right)+k_{1}+g_{1}\right]} \\
& \times\left(\begin{array}{cccc}
2 g_{1} & 0 & 2\left(g_{2}+1\right) & -2 g_{1} \\
2 k_{1} & 0 & -2\left(k_{2}+1\right) & -2 k_{1} \\
k_{1} g_{2}+k_{2} g_{1} & {\left[\left(k_{1} g_{2}+k_{2} g_{1}\right)+k_{1}+g_{1}\right]} & k_{2}-g_{2} & k_{1}+g_{1} \\
k_{1} g_{2}+k_{2} g_{1} & -\left[\left(k_{1} g_{2}+k_{2} g_{1}\right)+k_{1}+g_{1}\right] & k_{2}-g_{2} & k_{1}+g_{1}
\end{array}\right)
\end{aligned}
$$

The first column ${ }^{3}$ of this matrix is the weight vector for the potential solution. When multiplied by the potential vector this is identical to the field formula derived "by hand" from examination of the solution polynomial (sans space-charge).

\footnotetext{
${ }^{2}$ Use is made of the notation presented in Section 2.3 to simplify these examples.
} 


$$
\mathbf{w}=\frac{1}{2\left[\left(k_{1} g_{2}+k_{2} g_{1}\right)+k_{1}+g_{1}\right]}\left(\begin{array}{c}
2 g_{1} \\
2 k_{1} \\
k_{1} g_{2}+k_{2} g_{1} \\
k_{1} g_{2}+k_{2} g_{1}
\end{array}\right)=\frac{1}{C_{D}}\left(\begin{array}{c}
C_{N} \\
C_{S} \\
C_{E W} \\
C_{E W}
\end{array}\right)
$$

Turning to the source component of the solution, using the same four stencil points in the same order, the $\mathbf{Z}$ matrix and its transpose are shown below. Note this matrix is not square since four points are insufficient to determine the six coefficients that occur through $2^{\text {nd }}$ order (stencil symmetry eliminates only one coefficient). Also, the field solution errors occur at $4^{\text {th }}$ order and the equivalent source errors would be at $2^{\text {nd }}$ order. Hence, only the coefficients through $1^{\text {st }}$ order are included.

$$
\mathbf{Z}=\left(\begin{array}{rrr}
1 & 0 & 1 \\
1 & 0 & -1 \\
1 & 1 & 0 \\
1 & -1 & 0
\end{array}\right) \quad \mathbf{Z}^{\mathbf{T}}=\left(\begin{array}{cccc}
1 & 1 & 1 & 1 \\
0 & 0 & 1 & -1 \\
1 & -1 & 0 & 0
\end{array}\right)
$$

The product of $\mathbf{Z}$-transpose and $\mathbf{Z}$ produces a square matrix that can be inverted. Post multiplication by $\mathbf{Z}$-transpose then yields the generalized inverse of $\mathbf{Z}$.

$$
\mathbf{Z}^{\mathbf{T}} \mathbf{Z}=\left(\begin{array}{ccc}
4 & 0 & 0 \\
0 & 2 & 0 \\
0 & 0 & 2
\end{array}\right) \quad\left(\mathbf{Z}^{\mathbf{T}} \mathbf{Z}\right)^{-1}=\left(\begin{array}{ccc}
\frac{1}{4} & 0 & 0 \\
0 & \frac{1}{2} & 0 \\
0 & 0 & \frac{1}{2}
\end{array}\right) \quad\left(\mathbf{Z}^{\mathbf{T}} \mathbf{Z}\right)^{-1} \mathbf{Z}^{\mathbf{T}}=\left(\begin{array}{cccc}
\frac{1}{4} & \frac{1}{4} & \frac{1}{4} & \frac{1}{4} \\
0 & 0 & \frac{1}{2} & -\frac{1}{2} \\
\frac{1}{2} & -\frac{1}{2} & 0 & 0
\end{array}\right)
$$

The LTP source offset matrix is shown below where, again, offset terms defined in Section 2.3 above are used.

$$
\mathbf{Q}=\left(\begin{array}{ccc}
\frac{1}{2} k_{2} & 0 & \frac{1}{6} m_{1} \\
\frac{1}{2} g_{2} & 0 & -\frac{1}{6} n_{1} \\
0 & 0 & 0 \\
0 & 0 & 0
\end{array}\right)
$$

Right-multiplying $\mathbf{Q}$ by the generalized inverse of $\mathbf{Z}$ produces:

$$
\mathbf{Q}\left(\mathbf{Z}^{\mathbf{T}} \mathbf{Z}\right)^{-1} \mathbf{Z}^{\mathbf{T}}=\left(\begin{array}{cccc}
\frac{1}{8} k_{2}+\frac{1}{12} m_{1} & \frac{1}{8} k_{2}-\frac{1}{12} m_{1} & \frac{1}{8} k_{2} & \frac{1}{8} k_{2} \\
\frac{1}{8} g_{2}-\frac{1}{12} n_{1} & \frac{1}{8} g_{2}+\frac{1}{12} n_{1} & \frac{1}{8} g_{2} & \frac{1}{8} g_{2} \\
0 & 0 & 0 & 0 \\
0 & 0 & 0 & 0
\end{array}\right)
$$

3 The other columns are estimates for the weights of the other coefficients. These will be discussed later in conjunction with potential interpolation and electric field calculation. 
This matrix is now left-multiplied by the transpose of the weight vector, $\mathbf{w}^{\mathbf{T}}$, to generate the source weigh vector, $\omega$. Again, use has been made of terms defined above to simplify the expressions.

$$
\omega=\left[\mathbf{w}^{\mathbf{T}}\left\{\mathbf{Q}\left(\mathbf{Z}^{\mathrm{T}} \mathbf{Z}\right)^{-1} \mathbf{Z}^{\mathrm{T}}\right\}\right]^{\mathrm{T}}=\frac{1}{8 C_{D}}\left(\begin{array}{c}
C_{N}\left(k_{2}+\frac{2}{3} m_{1}\right)+C_{S}\left(g_{2}-\frac{2}{3} n_{1}\right) \\
C_{N}\left(k_{2}-\frac{2}{3} m_{1}\right)+C_{S}\left(g_{2}+\frac{2}{3} n_{1}\right) \\
C_{E W}\left(k_{2}+g_{2}\right) \\
C_{E W}\left(k_{2}+g_{2}\right)
\end{array}\right)
$$

The source contribution to the potential solution is determined by multiplying the source-weight and source vectors.

$$
\omega^{\mathrm{T}} \mathbf{S}=\frac{C_{E W}\left[S_{N}+S_{S}+\frac{1}{2}\left(k_{2}+g_{2}\right)\left(S_{E}+S_{W}\right)\right]+\frac{1}{3}\left(g_{1} m_{1}-k_{1} n_{1}\right)\left(S_{N}-S_{S}\right)}{4 C_{D}}
$$

Rearranging terms results in the expression below. This is very similar to the source terms in the potential solution formula derived "by hand" except for the term in brackets. Written in this manner, it is clear that this term represents an "average" using source values at surrounding points to estimate the source at the primary node, $S_{P}$. Note that inclusion of the $b_{11}$ space-charge term (far right term) occurs naturally in the matrix approach.

$$
\omega^{\mathrm{T}} \mathbf{S}=\frac{C_{E W}}{C_{D}}\left\{\frac{\left[S_{N}+S_{S}+\frac{1}{2}\left(k_{2}+g_{2}\right)\left(S_{E}+S_{W}\right)\right]}{4}\right\}+\frac{C_{\rho}\left(S_{N}-S_{S}\right)}{C_{D}}
$$

The potential formula derived from the matrix approach is shown below and is almost identical to the formula obtained by direct evaluation of the polynomial. The difference being use of the source value at the central point (direct) or an estimate for this value derived from the four stencil points (matrix). For this simple example, it is possible to unravel the connections and one form could be substituted for the other. In more complex cases, it is problematic whether unraveling such connections would be possible.

$$
U_{p}=\frac{C_{N} U_{N}+C_{S} U_{S}+C_{E W}\left(U_{E}+U_{W}\right)}{C_{D}}+\frac{C_{E W}\left\{S_{P}\right\}+C_{\rho}\left(S_{N}-S_{S}\right)}{C_{D}}
$$

This example demonstrates the equivalence of the "direct" and "matrix" approaches to deriving solution formulas. The advantage of the matrix approach is that it can be "automated" for use with larger and more complex (e.g. non-conformal) stencils.

We give a second example that includes the primary node. For the source polynomial, six points would be required in general to determine the coefficients through $b_{22}$. If the primary node is included, the uniform 5-point stencil is sufficient to determine all but the $b_{21}$ coefficient, which is eliminated by symmetry even in cylindrical coordinates. This will be illustrated below for a stencil with an aspect ratio of $v=2$. The $\mathbf{Z}$ matrix in this case is constructed as a square $5 \times 5$ matrix that includes the central node. 


$$
\mathbf{Z}=\left(\begin{array}{rrrrr}
1 & 0 & 0 & 0 & 0 \\
1 & 0 & 1 & 0 & 1 \\
1 & 0 & -1 & 0 & 1 \\
1 & 2 & 0 & 4 & 0 \\
1 & -2 & 0 & 4 & 0
\end{array}\right)
$$

Computing the inverse of $\mathbf{Z}$ and solving for the source-polynomial coefficients yields the matrix equation below. The coefficient formulas in this expression are standard finite difference relations.

$$
\left(\begin{array}{l}
b_{00} \\
b_{10} \\
b_{11} \\
b_{20} \\
b_{22}
\end{array}\right)=\left(\begin{array}{rrrrr}
1 & 0 & 0 & 0 & 0 \\
0 & 0 & 0 & 1 / 4 & -1 / 4 \\
0 & 1 / 2 & -1 / 2 & 0 & 0 \\
-1 / 4 & 0 & 0 & 1 / 8 & 1 / 8 \\
-1 & 1 / 2 & 1 / 2 & 0 & 0
\end{array}\right)\left(\begin{array}{c}
S_{P} \\
S_{N} \\
S_{S} \\
S_{E} \\
S_{W}
\end{array}\right)
$$

Normally, only the first three rows of the above relation would be used with the 5-point stencil to achieve full $2^{\text {nd }}$ order accuracy. The weight vector would have four elements, and $\mathbf{Q}$ would be a $4 \times 3$ matrix, as in the first example.

$$
\begin{gathered}
\mathbf{w}^{\mathbf{T}} \mathbf{Q}\left(\begin{array}{ccccc}
1 & 0 & 0 & 0 & 0 \\
0 & 0 & 0 & 1 / 4 & -1 / 4 \\
0 & 1 / 2 & -1 / 2 & 0 & 0
\end{array}\right)\left(\begin{array}{c}
S_{P} \\
S_{N} \\
S_{S} \\
S_{E} \\
S_{W}
\end{array}\right) \\
\frac{1}{C_{D}}\left(\begin{array}{c}
v^{2} C_{N} \\
v^{2} C_{S} \\
C_{E W} \\
C_{E W}
\end{array}\right)\left(\begin{array}{ccccc}
\frac{1}{2} k_{2} & \frac{1}{12} m_{1} & -\frac{1}{12} m_{1} & 0 & 0 \\
\frac{1}{2} g_{2} & -\frac{1}{12} n_{1} & \frac{1}{12} n_{1} & 0 & 0 \\
0 & 0 & 0 & 0 & 0 \\
0 & 0 & 0 & 0 & 0
\end{array}\right)\left(\begin{array}{c}
S_{P} \\
S_{N} \\
S_{S} \\
S_{E} \\
S_{W}
\end{array}\right) \\
\omega^{\mathbf{T}} \mathbf{S}=v^{2} \frac{1}{2}\left(g_{1} k_{2}+k_{1} g_{2}\right) S_{P}+\frac{1}{12}\left(g_{1} m_{1}-k_{1} n_{1}\right)\left[S_{N}-S_{S}\right] \\
C_{D}
\end{gathered}
$$

This expression for the source contribution to the potential is the same as that obtained by direct manipulation of the LTP.

\subsection{Interpolation of the Potential and Calculation of Electric Field}

Solving for the potential at off-node points and computing the electric field at on and off-node points requires determination of the LTP coefficients. The weight matrix derived above already 
includes values for potential LTP coefficients. These estimates can be used as is; however, one of the differences between interpolation and solution is that the primary node value is known and can be included in the computation. The matrix can be reconstructed to include the node potential, or, alternatively, its value can be subtracted from the other stencil points yielding a smaller matrix, as illustrated below.

$$
\left(\begin{array}{c}
\phi_{1}-\alpha_{10} U_{P} \\
\phi_{2}-\alpha_{20} U_{P} \\
\vdots \\
\phi_{L}-\alpha_{L 0} U_{P}
\end{array}\right)=\left(\begin{array}{cccc}
\alpha_{11} & \alpha_{12} & \ldots & \alpha_{1 M} \\
\alpha_{21} & \alpha_{22} & \mathrm{~K} & \alpha_{2 M} \\
\mathrm{M} & \mathrm{M} & \mathrm{O} & \mathrm{M} \\
\alpha_{L 1} & \alpha_{L 2} & \mathrm{~K} & \alpha_{L M}
\end{array}\right)\left(\begin{array}{c}
A_{1} \\
A_{2} \\
\mathrm{M} \\
A_{M}
\end{array}\right)
$$

However the matrices are constructed, determination of the coefficient vector proceeds similarly to the solution procedure developed above. Taking account of the possible non-square nature of the modified offset matrix, the solution is given by the expression below.

$$
\mathbf{A}=\left[\mathbf{Y}^{\mathbf{T}} \mathbf{Y}\right]^{-1} \mathbf{Y}^{\mathbf{T}} \Psi=\mathbf{M}^{\mathbf{T}} \Psi
$$

Different symbols have been used for these matrices as a reminder that they are modified from the solution matrices developed in previous sections.

$$
\begin{gathered}
\Psi=\left(\begin{array}{c}
\phi_{1}-\alpha_{10} U_{P} \\
\phi_{2}-\alpha_{20} U_{P} \\
\vdots \\
\phi_{L}-\alpha_{L 0} U_{P}
\end{array}\right) \quad \mathbf{Y}=\left(\begin{array}{cccc}
\alpha_{11} & \alpha_{12} & \ldots & \alpha_{1 M} \\
\alpha_{21} & \alpha_{22} & \ldots & \alpha_{2 M} \\
\mathrm{M} & \mathrm{M} & \mathrm{O} & \mathrm{M} \\
\alpha_{L 1} & \alpha_{L 2} & \mathrm{~K} & \alpha_{L M}
\end{array}\right) \quad \mathbf{A}=\left(\begin{array}{c}
A_{1} \\
A_{2} \\
\vdots \\
A_{M}
\end{array}\right) \\
\mathbf{M}=\mathbf{Y}\left[\mathbf{Y}^{\mathbf{T}} \mathbf{Y}\right]^{-1}
\end{gathered}
$$

Expanding this solution gives:

$$
\begin{aligned}
& \mathbf{A}=\mathbf{M}^{\mathbf{T}}\left(\mathbf{U}-\alpha_{0}^{\mathbf{T}} U_{P}\right)+\Theta^{\mathbf{T}} \mathbf{S} \\
& \Theta^{\mathbf{T}}=\mathbf{M}^{\mathbf{T}}\left[\mathbf{Q}\left(\mathbf{Z}^{\mathbf{T}} \mathbf{Z}\right)^{-1} \mathbf{Z}^{\mathbf{T}}\right]
\end{aligned}
$$

The LTP polynomial coefficients have now been determined and we are now in a position to compute the potential and electric field at off-node points. The relations for this, in matrix notation, can be written as shown below. The $\mathbf{M}, \Theta, \mathbf{Q}$ and $\mathbf{Z}$ matrices only need to be computed once (i.e. pre-computed), the potential and source vectors, $\mathbf{U}$ and $\mathbf{S}$, change during the course of a calculation, and the $\mathbf{x}_{i}$ and $\mathbf{q}_{i}$ are vectors of LTP offset terms at arbitrary points.

$$
\begin{gathered}
U_{i}\left(\varepsilon_{i}, \eta_{i}\right)=\mathbf{x}_{i}^{\mathbf{T}} \mathbf{A}-\mathbf{q}_{i}^{\mathbf{T}} \mathbf{B} \\
U_{i}=\mathbf{x}_{i}^{\mathbf{T}}\left[\mathbf{M}^{\mathbf{T}}\left(\mathbf{U}-\alpha_{0}^{\mathbf{T}} U_{P}\right)+\Theta^{\mathrm{T}} \mathbf{S}\right]-\mathbf{q}_{i}^{\mathbf{T}}\left(\mathbf{Z}^{\mathrm{T}} \mathbf{Z}\right)^{-1} \mathbf{Z}^{\mathrm{T}} \mathbf{S} \\
U_{i}=\mathbf{x}_{i}^{\mathbf{T}} \mathbf{M}^{\mathbf{T}}\left(\mathbf{U}-\alpha_{0}^{\mathrm{T}} U_{P}\right)+\left[\mathbf{x}_{i}^{\mathrm{T}} \mathbf{M}^{\mathrm{T}} \mathbf{Q}-\mathbf{q}_{i}^{\mathrm{T}}\right]\left(\mathbf{Z}^{\mathrm{T}} \mathbf{Z}\right)^{-1} \mathbf{Z}^{\mathrm{T}} \mathbf{S}
\end{gathered}
$$


The role of space charge in interpolation is complicated. Space charge effects have to be subtracted out to compute $\mathbf{A}$ and then added back in to interpolate.

These expressions are very general, hence the complexity of the resulting equations. These relations make no assumptions (other than continuity) concerning grid structure and they simplify somewhat for uniform grids. It is clear why low order interpolation is the norm in most particle codes.

In addition to the complexity, there are problems from a usage standpoint. The $\mathbf{U}$ and $\mathbf{S}$ vectors change during simulations. Hence, the interpolation vectors $\mathbf{A}$ and $\mathbf{B}$ have to be recomputed before each use. This makes high-order interpolation during a simulation, e.g. for integration of particle trajectories, computationally expensive for non-standard stencils (in either compute time and/or memory).

Electric fields can be computed by taking the partials of the field interpolation formula. Expressions for this are shown below for the general case, where $\hat{e}_{z}$ and $\hat{e}_{r}$ are unit vectors in the axial and radial directions respectively.

$$
\begin{gathered}
\mathbf{E}\left(\varepsilon_{i}, \eta_{i}\right)=-\frac{1}{h}\left(\hat{e}_{z} \frac{\partial U}{\partial \varepsilon}+\hat{e}_{r} \frac{\partial U}{\partial \eta}\right)_{i} \\
=-\left.\frac{1}{h}\left(\hat{e}_{z} \frac{\partial \mathbf{x}^{\mathbf{T}}}{\partial \varepsilon}+\hat{e}_{r} \frac{\partial \mathbf{x}^{\mathbf{T}}}{\partial \eta}\right)\right|_{i} \mathbf{A}+\left.\frac{1}{h}\left(\hat{e}_{z} \frac{\partial \mathbf{q}^{\mathbf{T}}}{\partial \varepsilon}+\hat{e}_{r} \frac{\partial \mathbf{q}^{\mathbf{T}}}{\partial \eta}\right)\right|_{i} \mathbf{B} \\
E_{r}\left(\varepsilon_{i}, \eta_{i}\right)=-\left.\frac{1}{h} \frac{\partial \mathbf{x}^{\mathbf{T}}}{\partial \eta}\right|_{i}\left[\mathbf{M}^{\mathbf{T}}\left(\mathbf{U}-\alpha_{0}^{\mathbf{T}} U_{P}\right)+\Theta^{\mathbf{T}} \mathbf{S}\right]+\left.\frac{1}{h} \frac{\partial \mathbf{q}^{\mathbf{T}}}{\partial \eta}\right|_{i}\left[\left(\mathbf{Z}^{\mathbf{T}} \mathbf{Z}\right)^{-1} \mathbf{Z}^{\mathbf{T}} \mathbf{S}\right] \\
E_{z}\left(\varepsilon_{i}, \eta_{i}\right)=-\left.\frac{1}{h} \frac{\partial \mathbf{x}^{\mathbf{T}}}{\partial \varepsilon}\right|_{i}\left[\mathbf{M}^{\mathbf{T}}\left(\mathbf{U}-\alpha_{0}^{\mathbf{T}} U_{P}\right)+\Theta^{\mathbf{T}} \mathbf{S}\right]+\left.\frac{1}{h} \frac{\partial \mathbf{q}^{\mathbf{T}}}{\partial \varepsilon}\right|_{i}\left[\left(\mathbf{Z}^{\mathbf{T}} \mathbf{Z}\right)^{-1} \mathbf{Z}^{\mathbf{T}} \mathbf{S}\right]
\end{gathered}
$$

The partials can be computed analytically. At the primary node, i.e. $\left(\varepsilon_{i}, \eta_{i}\right)=(0,0)$, this expression reduces to determination of the $a_{10}$ and $a_{11}$ LTP coefficients.

$$
\mathbf{E}(0,0)=-\frac{1}{h}\left(\hat{e}_{z} a_{10}+\hat{e}_{r} a_{11}\right)
$$

Using the 2-D cylindrical LTP developed in Section 2.3, the electric field to $2^{\text {nd }}$ order at a node in a square grid is given by the formulas below. While the axial field formula corresponds to the usual finite difference relation, the expression for the radial field differs considerably. The LTP treatment involves the potential at the central node and source effects in addition to the north and south potential values. Note that these additional terms trend to zero as the node radius increases toward infinity, i.e. as cylindrical approaches rectangular the usual expression is recovered.

$$
\begin{gathered}
E_{r}=-\frac{g_{2} U_{N}-k_{2} U_{S}+\left(k_{2}-g_{2}\right) U_{P}+\frac{1}{2}\left(g_{2} k_{2}-k_{2} g_{2}\right) S_{P}+\frac{1}{6}\left(g_{2} m_{1}+k_{2} n_{1}\right) b_{11}}{h\left(g_{2} k_{1}+k_{2} g_{1}\right)} \\
E_{z}=-\frac{U_{E}-U_{W}}{2 h}
\end{gathered}
$$




\subsection{Comments on Stencils and Coefficients}

An examination of the relationship between stencil properties and coefficient determination is warranted at this point. In general, all coefficients up through order $\mathrm{N}-1$ have to be determined or eliminated to develop an $N^{\text {th }}$ order formula, thus, seven separate points are required to guarantee so-called $2^{\text {nd }}$ order accuracy $\left(2^{\text {nd }}\right.$ order in the Laplace operator, but $4^{\text {th }}$ order in the potential). For the Poisson equation using a non-symmetric stencil, coefficients $a_{10}$ through $a_{31}$ have to be eliminated to determine $U_{P}$ to $2^{\text {nd }}$ order.

Stencils with geometric symmetry can lead to higher accuracy with fewer points provided the symmetry is reflected in the LTP as well. For the Cartesian Laplace equation with the square 5point stencil, the stencil has $N-S, E-W$ and diagonal symmetries, all of which are expressed in the LTP. Hence, only five points (including the primary node) are required to eliminate all coefficients up to $a_{40}$. However, in cylindrical coordinates the $N-S$ stencil symmetry is not reflected in the LTP and four points are insufficient to eliminate the $a_{31}$ coefficient. The $E$ - $W$ stencil symmetry is expressed in the LTP so terms that are odd in $\varepsilon$ and even in $\eta$ cancel out.

\subsection{Special Cases: On-Axis and First Row}

The cylindrical form of the Poisson PDE near the axis requires a different approach than used above. In this region $\delta$ would be infinite, so the original form of the PDE must be used. In this form since $r_{p}=0$, the $1 / r$ term becomes simply $1 /(h \eta)$. Also, the radial electric field on axis must be zero so the polynomial cannot have terms proportional to the first power of " $r$ ". With these restrictions, the PDE takes the normalized form shown below.

$$
\eta\left[\frac{\partial^{2} U}{\partial \varepsilon^{2}}+\frac{\partial^{2} U}{\partial \eta^{2}}+S\right]+\frac{\partial U}{\partial \eta}=0
$$

To ensure that the radial electric field is identically zero on-axis, the near-axis polynomial has to be modified to eliminate terms proportional to " $r$ ". This translates into all $a_{i 1}$ coefficients being identically zero, yielding the polynomial shown below. The usual routine of running this through the PDE, collecting terms and back substituting is applied to derive the LTP, which is shown to $2^{\text {nd }}$ order below.

$$
\begin{gathered}
U=\sum_{i=0}^{M} a_{i 0} \varepsilon^{i}+\sum_{i=2}^{M} \sum_{j=2}^{i} a_{i j} \varepsilon^{(i-j)} \eta^{j} \\
U=U_{P}+a_{10} \varepsilon+a_{20}\left(\varepsilon^{2}-\frac{1}{2} \eta^{2}\right)+a_{30} \varepsilon\left(\varepsilon^{2}-\frac{3}{2} \eta^{2}\right) \\
-\eta^{2}\left\{\frac{1}{4} b_{00}+\frac{1}{4} b_{10} \varepsilon+\frac{1}{9} b_{11} \eta\right\}
\end{gathered}
$$

The on-axis equivalent of the 5-point stencil in 2D provides only four points: east, west, north and the primary node. Evaluating the polynomial at the stencil points and eliminating coefficients provides the discrete solution formula below.

$$
U_{P}=\frac{E+W+4 N+S_{P}}{6}+\frac{2}{27} b_{11}
$$


Note that full $2^{\text {nd }}$ order requires compensating for the $b_{11}$ space charge term. The $b_{11}$ coefficient can be estimated from the source values at the stencil points. Referring to the source polynomial shown below, two expressions can be developed for computing $b_{11}$. The first is in principle $2^{\text {nd }}$ order with a $b_{22}$ leading error term and is sufficient for the "5-point" stencil. The second is more accurate with a leading error term involving $b_{33}$.

$$
\begin{gathered}
b_{11}=S_{N}-S_{P} \\
b_{11}=\frac{4 S_{N}-3 S_{P}-S_{N N}}{2}
\end{gathered}
$$

The potential solution formula on the first row above the axis also requires a special LTP formula. Recall the comment above concerning the analytic term $C_{1}(z)$ when $z=-1$, this is the situation on the first row. The second order solution formula derived from the near-axis LTP is somewhat unusual. It does not involve the east and west points, only the on-axis (south) and north points. To obtain full $2^{\text {nd }}$ order the $b_{11}$ space charge coefficient must be included. This also involves only the south, north and central node points.

$$
U_{P}=\frac{3 U_{S}+U_{N}}{4}+\frac{4 S_{P}-3 S_{S}-S_{N}}{18}
$$

On the axis the radial electric field is identically zero. The axial electric field is straightforward and given by the standard formula.

$$
E_{z}(0,0)=\frac{U_{W}-U_{E}}{2 h} \quad E_{r}(0,0)=0
$$

For the first row of nodes above the cylindrical axis, the electric field formulas can be derived using the near-axis polynomial. The axial electric field is, again, straightforward and given by the standard formula. The radial electric field based on the near-axis LTP is shown below.

$$
E_{r}(0,1)=\frac{9 U_{S}-U_{N}-8 U_{P}}{6 h}
$$

\section{LTP on Non-Uniform Grids}

There are numerous physical problems with multiple scale lengths that make the use of uniform grids computationally expensive, hence the need for accurate solution techniques on nonuniform grids. The LTP polynomial is not dependent on any particular grid shape or spacing and can be adapted to yield solution formulas for non-uniform grids in a straightforward manner.

\subsection{Non-Uniform Grid in 1-D}

Returning to the 1-D LTP and using only the relevant terms, we can evaluate this polynomial at arbitrary offsets from the primary node. We will leave " $h$ " indefinite for the moment and label the neighboring grid points $\eta_{N}$ and $-\eta_{S}$, yielding the expressions below. 


$$
\begin{aligned}
& U_{N}=U_{P}+\eta_{N} C_{1}\left(\delta \eta_{N}\right) a_{1}-\eta_{N}^{2} \frac{C_{2}\left(\delta \eta_{N}\right)}{2} b_{0}-\eta_{N}^{3} \frac{C_{3}\left(\delta \eta_{N}\right)}{6} b_{1} \\
& U_{S}=U_{P}-\eta_{S} C_{1}\left(-\delta \eta_{S}\right) a_{1}-\eta_{S}^{2} \frac{C_{2}\left(-\delta \eta_{S}\right)}{2} b_{0}+\eta_{S}^{3} \frac{C_{3}\left(-\delta \eta_{S}\right)}{6} b_{1}
\end{aligned}
$$

These can be combined to produce a discrete approximation for the potential at the primary node.

$$
\begin{aligned}
U_{P}= & \frac{\eta_{S} C_{1}\left(-\delta \eta_{S}\right) U_{N}+\eta_{N} C_{1}\left(\delta \eta_{N}\right) U_{S}}{\left[\eta_{S} C_{1}\left(-\delta \eta_{S}\right)+\eta_{N} C_{1}\left(\delta \eta_{N}\right)\right]} \\
& +\frac{\eta_{N} \eta_{S}\left[\eta_{N} C_{1}\left(-\delta \eta_{S}\right) C_{2}\left(\delta \eta_{N}\right)+\eta_{S} C_{1}\left(\delta \eta_{N}\right) C_{2}\left(-\delta \eta_{S}\right)\right] S_{P}}{2\left[\eta_{S} C_{1}\left(-\delta \eta_{S}\right)+\eta_{N} C_{1}\left(\delta \eta_{N}\right)\right]} \\
& +\frac{\left[\eta_{N}^{2} C_{1}\left(-\delta \eta_{S}\right) C_{3}\left(\delta \eta_{N}\right)-\eta_{S}^{2} C_{1}\left(\delta \eta_{N}\right) C_{3}\left(-\delta \eta_{S}\right)\right]}{6\left[\eta_{S} C_{1}\left(-\delta \eta_{S}\right)+\eta_{N} C_{1}\left(\delta \eta_{N}\right)\right]} \frac{\eta_{S}^{2} S_{N}-\eta_{N}^{2} S_{N}+\left(\eta_{N}^{2}-\eta_{S}^{2}\right) S_{P}}{\left(\eta_{N}+\eta_{S}\right)}
\end{aligned}
$$

Note that the source terms have a hidden $h^{2}$ dependence that comes from the definition of $S$.

As written, this relation appears more complicated than it really is. The matrix formulation presented above provides a systematic technique for solving general cases in 2-D and 3-D.

\subsection{Non-Uniform Grids in 2-D}

In two and three dimensions, simulation codes often make use of non-uniform, but orthogonal, rectangular grids in order to better match boundaries. Such meshes can be denoted by independent functions in the two coordinates.

$$
x_{i}=f\left(x_{0}, \Delta x_{0}, i\right) \quad y_{i}=g\left(y_{0}, \Delta y_{0}, i\right)
$$

Hence, for any particular node $\left(x_{i}, y_{i}\right)$ the distances to neighboring nodes will not necessarily be equal. We arbitrarily choose the east arm length to be the stencil metric $h$ and the other arm lengths are expressed in terms of $h$. Proceeding counterclockwise around the stencil, the other arm lengths will be denoted as $h_{1} h, h_{2} h$ and $h_{3} h$, where $h_{\mathrm{n}}$ is a unit-less multiplier.

It is well known that for a 5-point stencil with unequal arm lengths only first order accuracy can be obtained. Now, seven independent points (in addition to the primary node) are required for $2^{\text {nd }}$ order accuracy in the general case. Making use of the corner points in a non-uniform, orthogonal mesh permits this condition to be satisfied with a total of eight stencil points. The $8^{\text {th }}$ point means that the generalized inverse should be used rather than arbitrarily eliminating one point to form a square matrix. Below we give an example using the Cartesian Poisson LTP, shown to $2^{\text {nd }}$ order below.

$$
\begin{aligned}
U(\varepsilon, \eta) & =a_{00}+a_{10} \varepsilon+a_{11} \eta+a_{20}\left(\varepsilon^{2}-\eta^{2}\right)+a_{21} \varepsilon \eta \\
& +a_{30} \varepsilon\left(\varepsilon^{2}-3 \eta^{2}\right)+a_{31} \eta\left(\varepsilon^{2}-\frac{1}{3} \eta^{2}\right) \\
& -\frac{1}{2}\left[b_{00} \eta^{2}+b_{10} \varepsilon \eta^{2}+\frac{1}{3} b_{11} \eta^{3}\right]
\end{aligned}
$$


This polynomial is valid around the primary node provided the region is homogeneous. The stencil points for a non-uniform, orthogonal rectangular mesh are then given by:

$$
\begin{array}{llll}
E=(1,0) & N=\left(0, h_{1}\right) & W=\left(-h_{2}, 0\right) & S=\left(0,-h_{3}\right) \\
N E=\left(1, h_{1}\right) & N W=\left(-h_{2}, h_{1}\right) & S W=\left(-h_{2},-h_{3}\right) & S E=\left(1,-h_{3}\right)
\end{array}
$$

The potential offset matrix for this stencil is given below.

$$
\mathbf{X}=\left(\begin{array}{ccccccc}
1 & 1 & 0 & 1 & 0 & 1 & 0 \\
1 & 0 & h_{1} & -h_{1}^{2} & 0 & 0 & -\frac{1}{3} h_{1}^{3} \\
1 & -h_{2} & 0 & h_{2}^{2} & 0 & -h_{2}^{3} & 0 \\
1 & 0 & -h_{3} & -h_{3}^{2} & 0 & 0 & \frac{1}{3} h_{3}^{3} \\
1 & 1 & h_{1} & 1-h_{1}^{2} & h_{1} & 1-3 h_{1}^{2} & h_{1}\left(1-\frac{1}{3} h_{1}^{2}\right) \\
1 & -h_{2} & h_{1} & h_{2}^{2}-h_{1}^{2} & -h_{1} h_{2} & -h_{2}\left(h_{2}^{2}-3 h_{1}^{2}\right) & h_{1}\left(h_{2}^{2}-\frac{1}{3} h_{1}^{2}\right) \\
1 & -h_{2} & -h_{3} & h_{2}^{2}-h_{3}^{2} & h_{2} h_{3} & -h_{2}\left(h_{2}^{2}-3 h_{3}^{2}\right) & -h_{3}\left(h_{2}^{2}-\frac{1}{3} h_{3}^{2}\right) \\
1 & 1 & -h_{3} & 1-h_{3}^{2} & -h_{3} & 1-3 h_{3}^{2} & -h_{3}\left(1-\frac{1}{3} h_{3}^{2}\right)
\end{array}\right)
$$

The solution weights would be obtained from the first column of the generalized inverse of $\mathbf{X}$.

$$
\mathbf{W}=\left[\mathbf{X}\left(\mathbf{X}^{\mathrm{T}} \mathbf{X}\right)^{-1}\right]
$$

Since the form of the offset matrix is known, it can be constructed quickly and inverted numerically. The solution weights do not change unless the mesh is modified, so they can be precomputed and stored. This would significantly increase storage requirements since eight weights would be required at each node in the general case.

Inclusion of space charge in the field solve occurs in exactly the same manner as outlined in the matrix development above. There is a temptation, because of the number of stencil points, to include more space charge coefficients. However, the field solve is only $2^{\text {nd }}$ order accurate, which the $b_{00}, b_{10}$ and $b_{11}$ space charge coefficients are sufficient to maintain. The additional points can be used to determine these coefficients more accurately, but higher coefficients should not be included.

The space-charge polynomial offset matrix can be constructed in several ways: 1) use only the 5point star, 2) use all 9 points or 3) use all 9 points and six coefficients but only include the three necessary coefficients in the field calculation. It is very tempting to use the first option. Especially since in the regular-grid case only the charge at the primary node is required for $2^{\text {nd }}$ order accuracy (in Cartesian coordinates). The space-charge offset matrices for all options are shown below in order. 


$$
\begin{aligned}
& \mathbf{Z}=\left(\begin{array}{ccc}
1 & 0 & 0 \\
1 & 1 & 0 \\
1 & 0 & h_{1} \\
1 & -h_{2} & 0 \\
1 & 0 & -h_{3}
\end{array}\right) \\
& \mathbf{Z}=\left(\begin{array}{ccc}
1 & 0 & 0 \\
1 & 1 & 0 \\
1 & 0 & h_{1} \\
1 & -h_{2} & 0 \\
1 & 0 & -h_{3} \\
1 & 1 & h_{1} \\
1 & -h_{2} & h_{1} \\
1 & -h_{2} & -h_{3} \\
1 & 1 & -h_{3}
\end{array}\right) \quad \mathbf{Z}=\left(\begin{array}{cccccc}
1 & 0 & 0 & 0 & 0 & 0 \\
1 & 1 & 0 & 1 & 0 & 0 \\
1 & 0 & h_{1} & 0 & 0 & h_{1}^{2} \\
1 & -h_{2} & 0 & h_{2}^{2} & 0 & 0 \\
1 & 0 & -h_{3} & 0 & 0 & h_{3}^{2} \\
1 & 1 & h_{1} & 1 & h_{1} & h_{1}^{2} \\
1 & -h_{2} & h_{1} & h_{2}^{2} & -h_{2} h_{1} & h_{1}^{2} \\
1 & -h_{2} & -h_{3} & h_{2}^{2} & h_{2} h_{3} & h_{3}^{2} \\
1 & 1 & -h_{3} & 1 & -h_{3} & h_{3}^{2}
\end{array}\right)
\end{aligned}
$$

The space charge weight matrix is then formed as shown in the matrix section. For the second form of $\mathbf{Z}$, one would only use the top three rows in computing the space-charge contribution to the field solution.

$$
\Omega^{\mathrm{T}}=\mathbf{W}^{\mathrm{T}} \mathbf{Q}\left(\mathbf{Z}^{\mathrm{T}} \mathbf{Z}\right)^{-1} \mathbf{Z}^{\mathrm{T}}
$$

The space-charge component of the LTP is contained in the matrix $\mathbf{Q}$, shown below. Note that there is a row in the $\mathbf{Q}$ matrix for every stencil point, the eight non-primary nodes in this case. Keep in mind that $\mathbf{Q}$ is a component of the LTP and not the polynomial that directly describes the source distribution. Therefore, this does not require using the source at every stencil point, it requires knowing the necessary number of source polynomial coefficients.

$$
\mathbf{Q}=\frac{1}{2}\left(\begin{array}{ccc}
0 & 0 & 0 \\
h_{1}^{2} & 0 & \frac{1}{3} h_{1}^{3} \\
0 & 0 & 0 \\
h_{3}^{2} & 0 & -\frac{1}{3} h_{3}^{3} \\
h_{1}^{2} & h_{1}^{2} & \frac{1}{3} h_{1}^{3} \\
h_{1}^{2} & -h_{2} h_{1}^{2} & \frac{1}{3} h_{1}^{3} \\
h_{3}^{2} & -h_{2} h_{3}^{2} & -\frac{1}{3} h_{3}^{3} \\
h_{3}^{2} & h_{3}^{2} & -\frac{1}{3} h_{3}^{3}
\end{array}\right)
$$

The field solution would be based on the full expression incorporating the field and space-charge components. Again, the basic matrix relation was developed above and remains valid here. 


$$
U_{p}=\mathbf{w}^{\mathbf{T}} \mathbf{U}+\omega^{\mathbf{T}} \mathbf{S}
$$

Here $\mathbf{w}$ and $\boldsymbol{\omega}$ are $8 \times 1$ vectors, the first columns of the $\mathbf{W}$ and $\Omega$ matrices. The elements of $\mathbf{S}$ at the stencil points are related to the usual charge density values by $S=4 \pi h^{2} \rho$. The " $h$ " is the actual length of the east arm and it converts the area scale from $\mathrm{cm}^{2}$ to cell ${ }^{2}$.

The same basic approach should be applied in the cylindrical Poisson case. The offset matrices would be those appropriate to cylindrical geometry. The inversion process, etc. would be the same as above.

\subsection{Higher Resolution Grid Patches}

Another approach used in particle codes to provide high resolution with minimal computational costs is the use of grid patches, i.e. a small region of high resolution mesh embedded in the larger mesh (usual a factor of 2 in mesh size). This is illustrated in the figure below where the primary grid is represented by black lines and the grid patch by red lines. There are two nodes, marked with an " $X$ " that require special treatment. For each of these nodes, the available stencil points (a total of 7) are marked with blue circles. Using the LTP matrix approach, deriving solution weights would be straightforward. The number and placement of stencil points are sufficient to ensure $2^{\text {nd }}$ order solution accuracy.

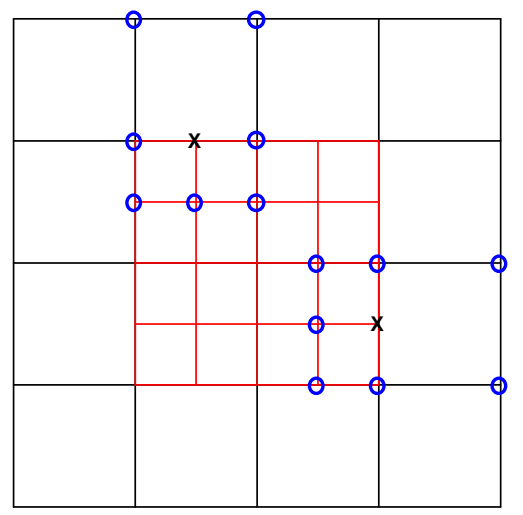

Figure 2: Schematic illustration of a grid "patch."

\section{Implementation of The LTP Technique}

\subsection{Implementation and Testing of the 1-D Cylindrical LTP}

The 1-D cylindrical LTP formulas presented above were implemented in a Fortran code for testing. For expediency, an iterative, even-odd (checkerboard) SOR routine was used for the potential solutions. LTP is fully compatible with more advanced solvers and a direct matrix inversion (via MatLab) was used for testing in 2-D. Standard cylindrical finite difference formulas were also programmed for comparison in both the 1-D and 2-D cases. Both LTP and FD were implemented in versions for explicitly uniform-grids and for non-uniform grids.

Several analytic source and potential solutions were programmed for testing. A few of the cases were not compatible with inclusion of the axis due to an infinity at $r=0$ for either the source or potential function. Table 4.1 below shows the source and potential formulas for each case. For 
the coaxial Case-7 and cylindrical Case- $8, R_{1}$ is the inner radius with associated potential $V_{1}$, and $R_{2}$ is the outer radius with associated potential $V_{2}$. For the $\operatorname{sinc}(x)$ source in Case- $9, r_{\mathrm{b}}$ is the outer source radius, $R$ the outer cylindrical radius and $S_{\mathrm{o}}$ the source amplitude.

Table 4.1: Analytic Source and Potential Formulas

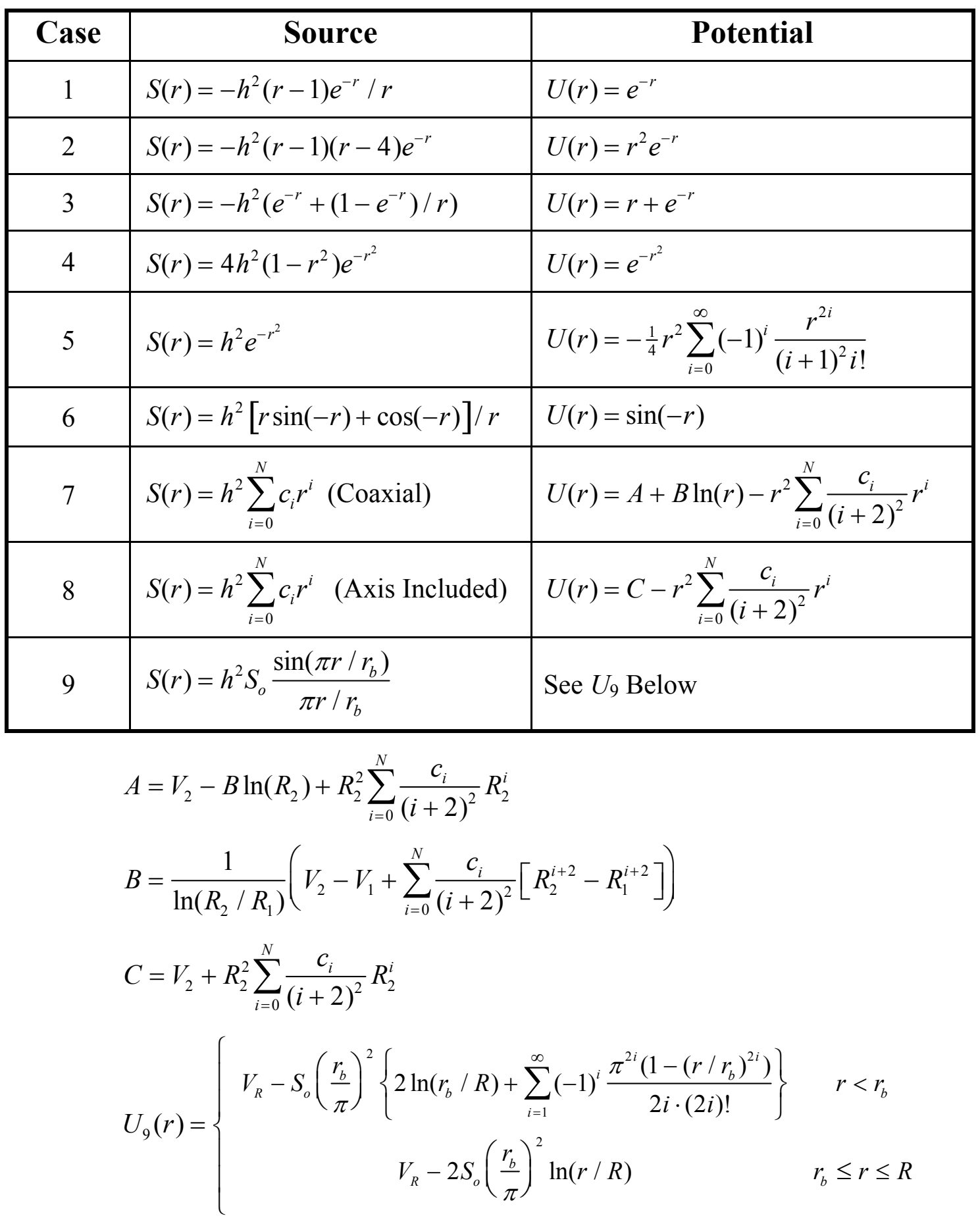

In addition to testing LTP with different source distributions, performance on different grids was examined. For the 1-D tests, three types of grids were programmed: 1) uniform, 2) random-shift and 3 ) constant $\Delta U$. The random-shift grid was generated by shifting each uniform-grid node by 
a random fraction of $h$. The constant $\Delta U$ grid was generated by adjusting node spacing to yield a constant potential difference between nodes. These grids are shown below for a coaxial $4^{\text {th }}$ degree polynomial source (Case-7), Fig. 3, and a sinc source (Case-9), Fig. 4.
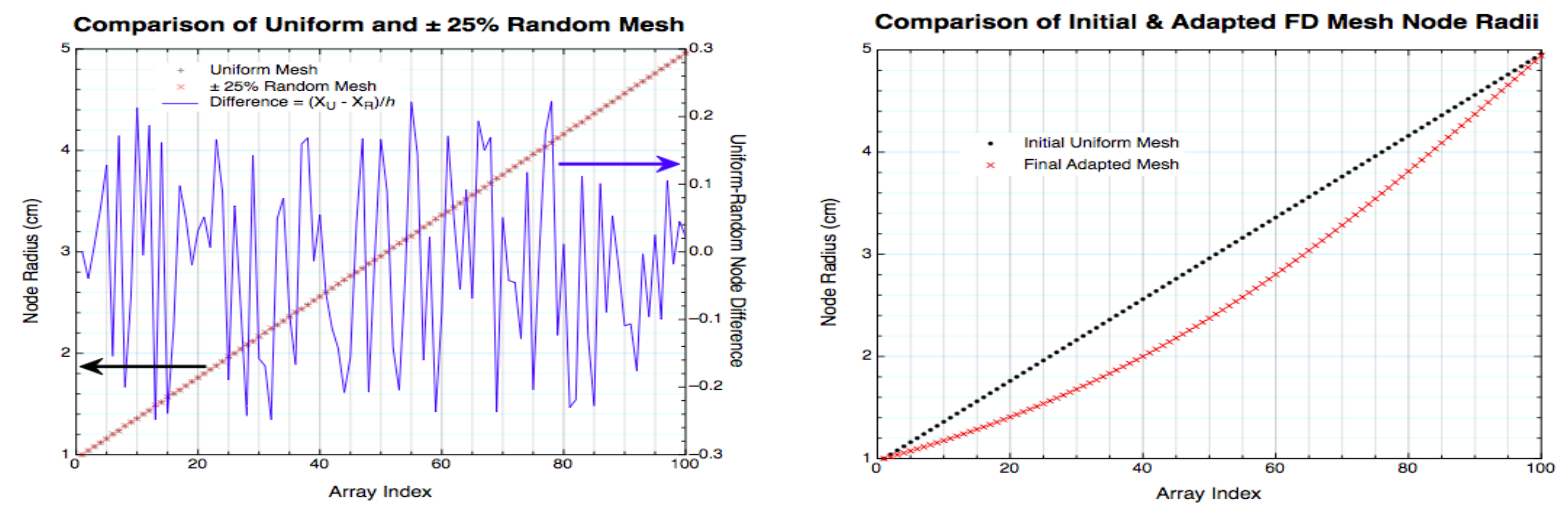

Figure 3: Grids for coaxial Case-7 with $4^{\text {th }}$ degree polynomial source. The left-hand plot shows node radii for the uniform (black) and random (red) grids. The blue curve shows random-grid node separation normalized to $h$. The right-hand plot shows the node radii for the uniform (black) and constant $\Delta U$ (red) grids. The nodes are tighter near the inner radius and further apart at the outer.
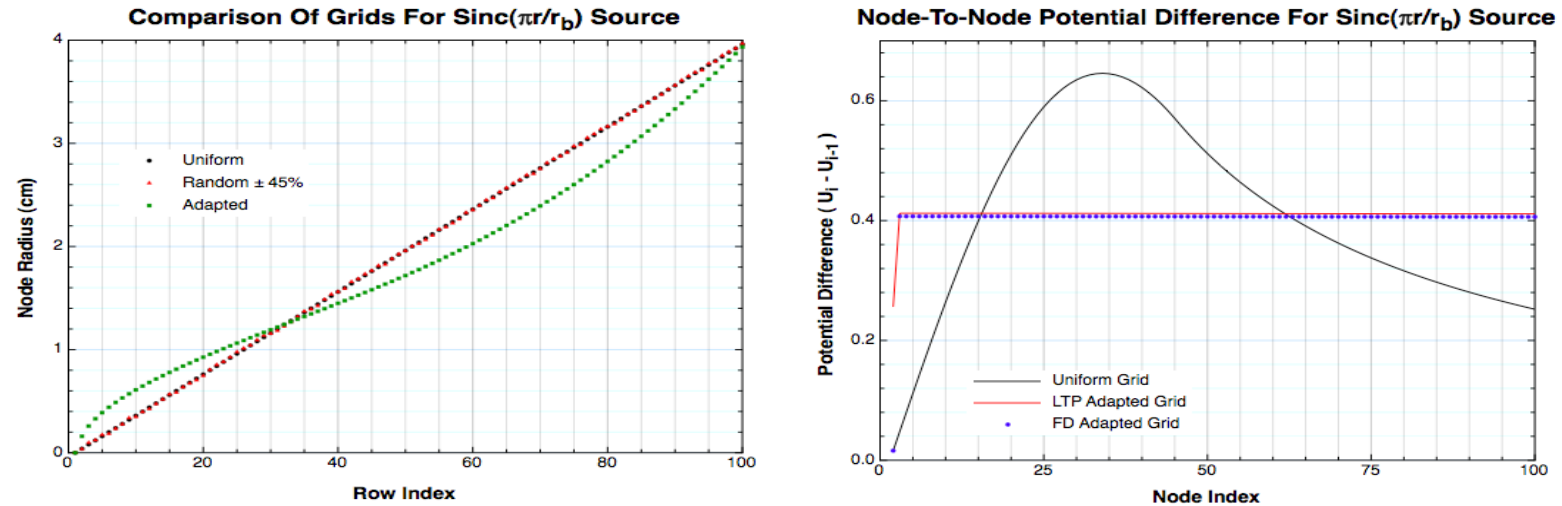

Figure 4: Grids for sinc source Case-9. The left-hand plot shows node radii for the uniform (black), random (red) and constant $\Delta U$ (green) grids. The right-hand plot shows the node-to-node potential difference for the uniform (black) and constant $\Delta U$ (red and blue) grids. Here, the nodes cluster around the outer source edge.

Convergence with grid spacing was one property that was checked in both the 1-D and 2-D tests. Convergence for the sinc source (Case-9) with the constant $\Delta U$ grid is shown in Fig. 5 for both LTP and FD. As the figure shows, convergence for both formulations is roughly $2^{\text {nd }}$ order. At smaller grid spacing, LTP has $3^{\text {rd }}$ order convergence. On average, in all of the test cases, LTP was at least $2^{\text {nd }}$ order or better. In contrast, FD showed slightly less than $2^{\text {nd }}$ order convergence in some cases and never showed faster convergence than LTP. Usually, both methods showed essentially $2^{\text {nd }}$ order convergence whether the grid was uniform or not ${ }^{4}$.

${ }^{4}$ We expected less than $2^{\text {nd }}$ order convergence in the non-uniform cases due to $1^{\text {st }}$ order truncation errors on such grids. We have no explanation for this result at present but speculate it may be an artifact of 1-D. Comparable 2-D calculations have not yet been performed. 


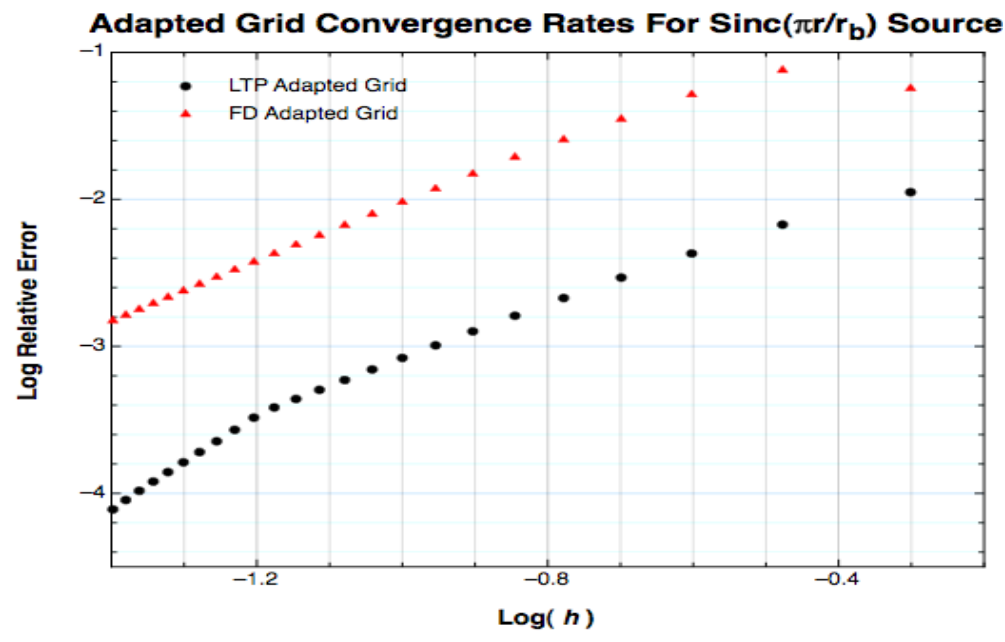

Figure 5: $\log$ of the relative potential error versus the $\log$ of the average grid spacing for the sinc source with constant $\Delta U$ grid for LTP (black) and finite difference (red). Both start out roughly $2^{\text {nd }}$ order, as expected, but the LTP convergence slope becomes $3^{\text {rd }}$ order at smaller grid spacing.

Details differed from case to case, but the results presented below for the two source functions, Case-7 and Case-9, provide a representative overview of LTP performance. The first case, the Case-7 coaxial $4^{\text {th }}$ degree polynomial source, does not include the axis. The plots in Fig. 6 show the relative error in the numerical potential, i.e. err $=\log \left|\left(U_{\text {analytic }}-U_{\text {numeric }}\right) / U_{\text {analytic }}\right|$, versus radius. The left-hand graph compares LTP and FD relative errors on a uniform grid. The center and right plots compare LTP results on a uniform grid versus LTP on a $\pm 25 \%$ random-shift grid (center) and a constant $\Delta U$ grid (right).

LTP shows significantly lower error than FD in this case, especially near the axis. Some cases showed even better LTP performance, as much as 3-4 orders of magnitude, while others showed smaller differences. In a few cases, LTP had lower errors at small radii while FD was better at larger radii, as seen in the next case. In the cases examined in this project, FD never showed significantly lower overall error than LTP. The two formulations were either comparable or LTP was significantly better.
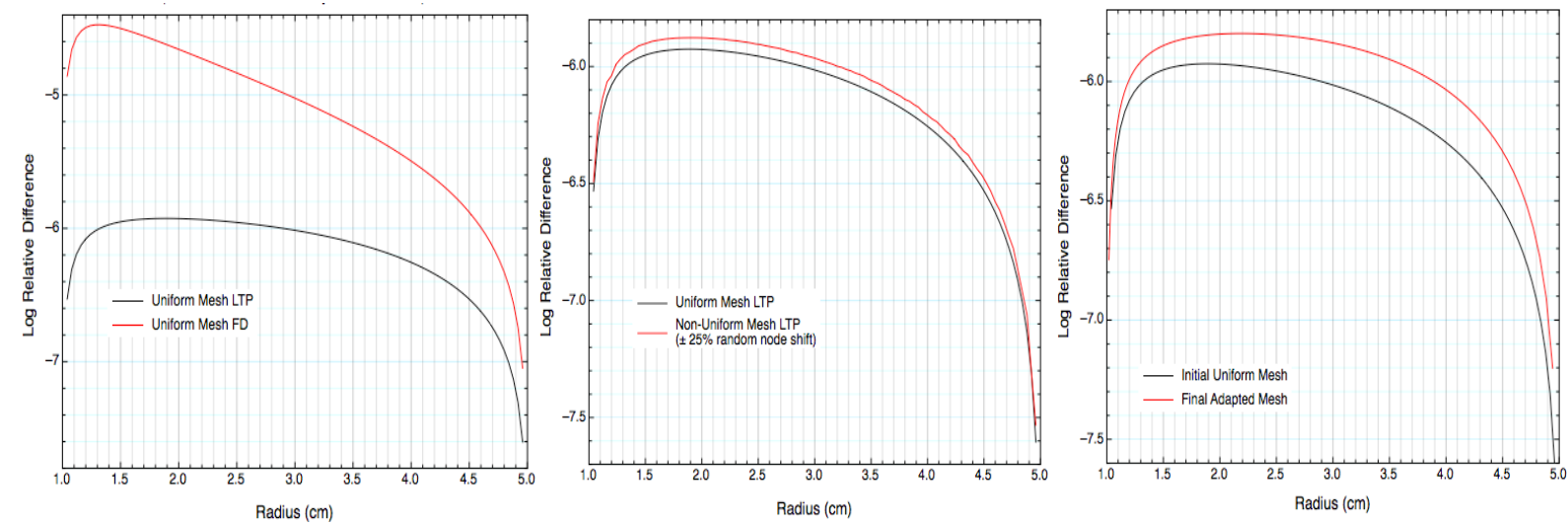

Figure 6: Relative error $(\log )$ of the computed potential versus radius for the Case-7 $4^{\text {th }}$ degree polynomial source. The right-hand plot compares LTP and FD errors. The center plot compares LTP errors on uniform and randomshift grids. The right-hand plot compares LTP errors on uniform and constant $\Delta U$ grids. Note the relatively small shifts in error and the smoothness of the transitions in the two non-uniform grid cases. 
Although not explicitly shown in Fig. 6, LTP appeared to be more robust to perturbed configurations. The solution errors in the random-shift and constant $\Delta U$ grids in Fig. 6 are relatively small and show smooth transitions from the uniform grid error. Compare the LTP curves in Fig. 6 with the corresponding LTP and FD curves in Fig. 7. Note the lower magnitude and relative uniformity of the LTP error shifts compared to FD. In general, FD error shifts for non-uniform grids were larger and showed more variation than did the LTP results.
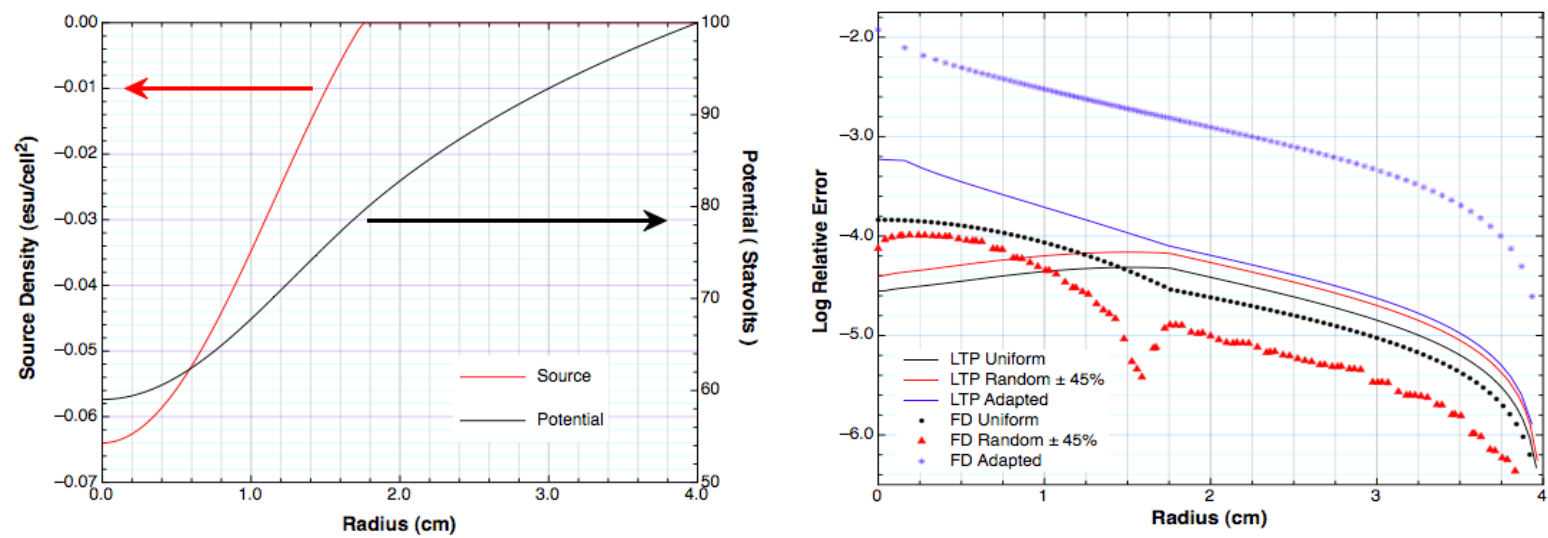

Figure 7: Results for the Case-9 sinc source. The left-hand plot shows the source function (red) and associated analytic potential function (black). The right-hand plot shows the relative error $(\log )$ in the computed potential versus radius for both LTP and FD solutions on all three grids. Surprisingly for the FD solver, the specific random grid in this calculation showed lower error than the uniform grid solution, and the lowest error overall at higher radii.

An analytic example with some of the features of a photoinjector charge bunch was desired, so a sinc source function was programmed. The sinc source has peak magnitude on the axis, a first derivative that is zero on-axis, a finite radius and an analytic potential solution. The functions for the sinc source and potential are listed in Table 4.1 (Case-9) and shown in the left-hand plot in Fig. 7. Implemented in this fashion, the sinc source has a somewhat abrupt termination at $r=r_{b}$.

Relative error for LTP and FD numerical solutions for the sinc source are shown in the righthand plot in Fig. 7. On the uniform grid, the LTP solution has lower error until near the source edge, where FD becomes slightly better. The abrupt end of the source might, at least partially, explain this result. Numerical calculation of the $b_{1}$ coefficient uses source values at three nodes. Because the source terminates so suddenly, errors will occur in calculating $b_{1}$ in the vicinity of the source edge. These errors propagate to the rest of the nodes and increase the overall solution error. Note that the peak LTP error occurs at the source edge. Since there is no " $b_{1}$ " in the FD formula, this is not an issue for the FD solution.

The LTP solutions on the non-uniform grids have slightly larger errors, except the constant $\Delta U$ grid, which shows a larger jump in error in the source region. The error jump for FD with the constant $\Delta U$ grid is larger still, by an order of magnitude, and occurs over the full radial range. A surprising result involves the FD solution on the random-shift grid, which displays lower error than the uniform grid result and the lowest error of all at higher radii. This particular randomshift node distribution produces an error profile that flips sign, i.e. goes through zero, at a radius close to the source edge. The sign flip reduces the overall error. (Wonderful, if such grids could be programmed in advance and on purpose!)

The results of the 1-D cylindrical Poisson PDE tests can be summarized as follows: 
- $\quad$ LTP produces noticeably smaller errors than FD for most cases

- $\quad$ LTP has $2^{\text {nd }}$ order convergence, or better, versus grid size

- LTP produces more accurate results near the axis than FD

- LTP is extremely accurate for coaxial configurations

- LTP is more robust than FD on non-uniform grids

- The $b_{1}$ coefficient requires careful evaluation in regions with source discontinuities

\subsection{MATLAB 2-D Implementation and Testing}

A 2-D code in r-z demonstrating the superiority of the LTP algorithm near the axis for Gaussian beams was implemented using MATLAB. The system included $\mathrm{r}$ from 0 to 4 and $\mathrm{z}$ from -2 to 2 . The number of cells used in each direction varied from 100 to 800 . The source function used was a Gaussian, $\exp \left(-r^{2}\right)$. Two different boundary conditions were applied, a periodic condition on the left and right radial boundaries, or a Dirichlet condition on the radial boundaries.

In the first case, the potential values at the ends of the cylinder are assumed to be identical. The results comparing the LTP and FD methods using 800 cells are shown below. The source function and the corresponding potential are shown below in Fig. 8. The absolute error for the LTP and FD methods for the periodic case are shown Fig. 9. The convergence graph for the case is shown below in the left panel of Fig. 12. The results clearly indicate the superiority of the LTP method by an order of magnitude.
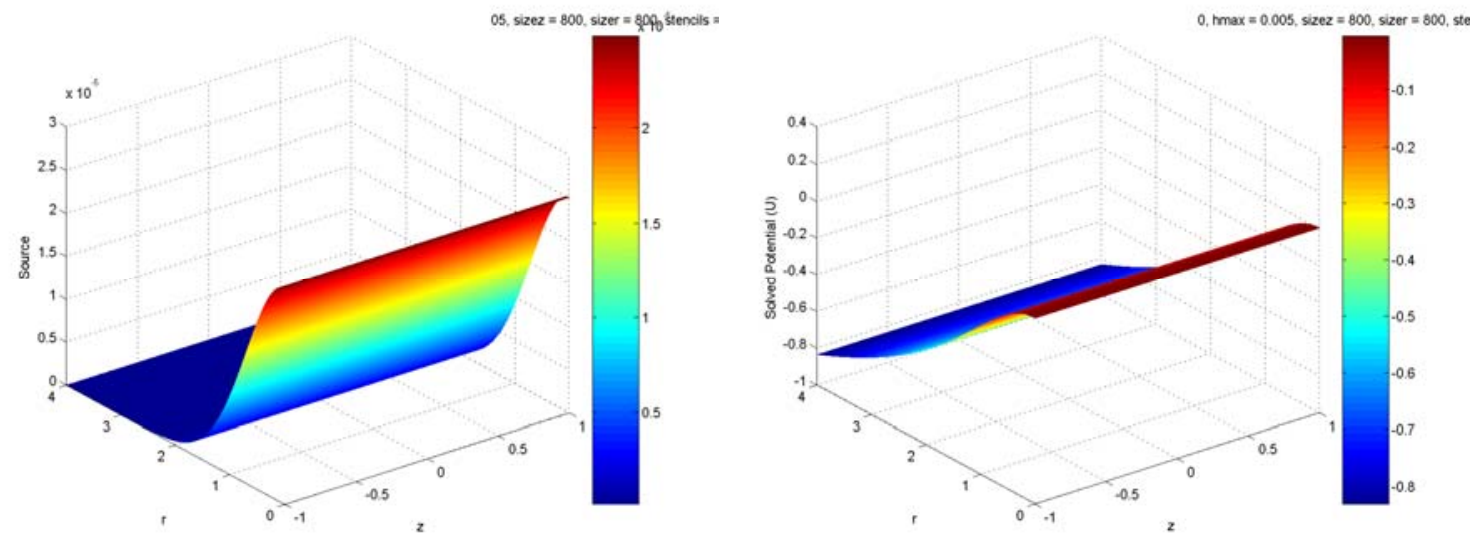

Figure 8: Source function $\mathrm{S}=\exp \left(-r^{2}\right)$ (left) and analytic solution to Poisson's equation (right) for the periodic case. 

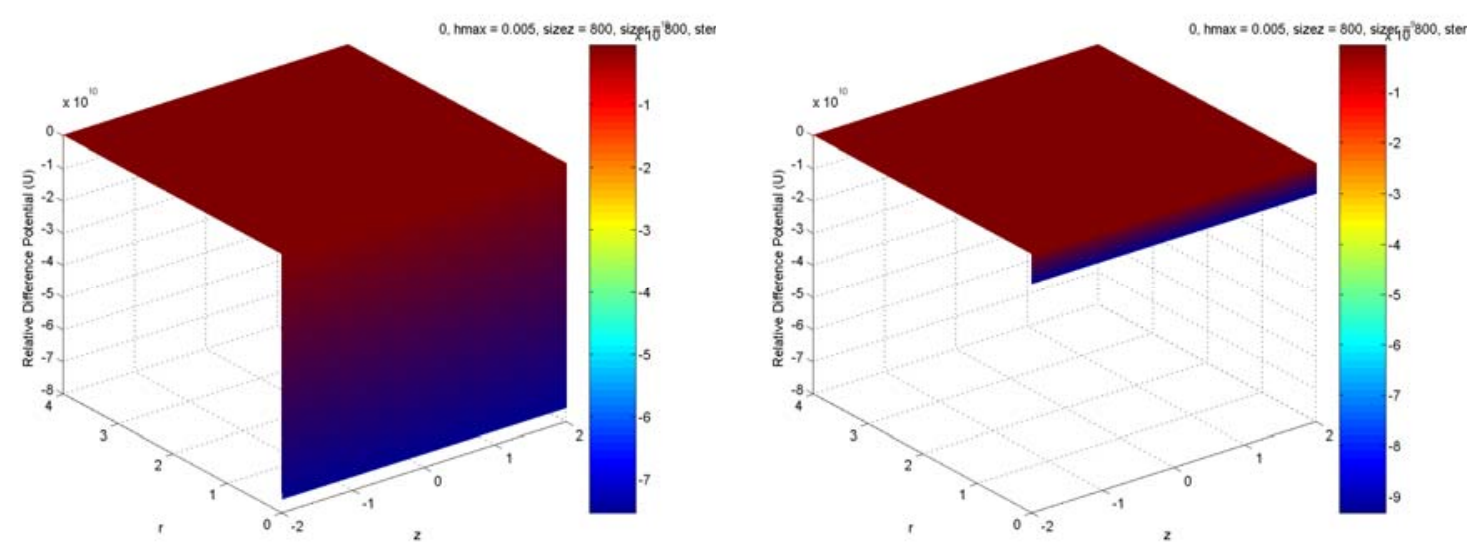

Figure 9: Relative error for the FD (left) and LTP (right) schemes for an 800x800 cell mesh for the periodic case.

In the second case, the boundary conditions for the potential are taken to be the analytic potential values. The results comparing the LTP and FD methods using 800 cells are shown below. The source function and analytic solution are shown in Fig 10. The absolute error for the FD and LTP methods are shown in Fig. 11. Again, the results clearly indicate the superiority of the LTP method by about an order of magnitude.
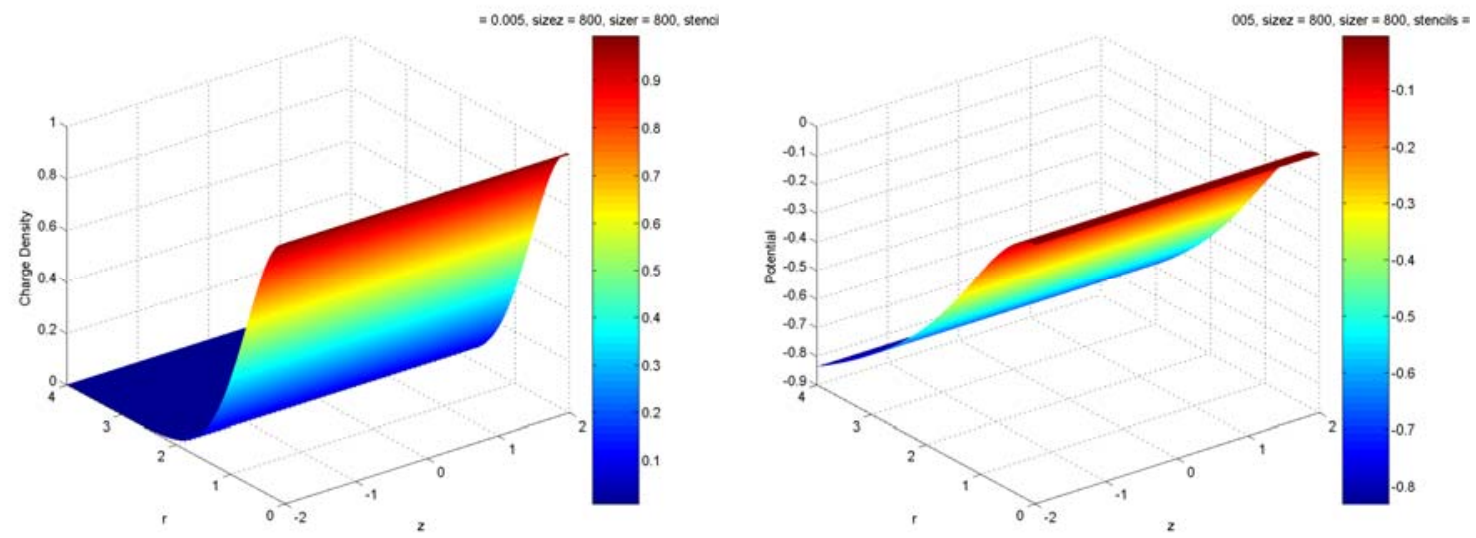

Figure 10: Source function $\mathrm{S}=\exp \left(-r^{2}\right)$ (left) and analytic solution to Poisson's equation (right) for the bounded case.
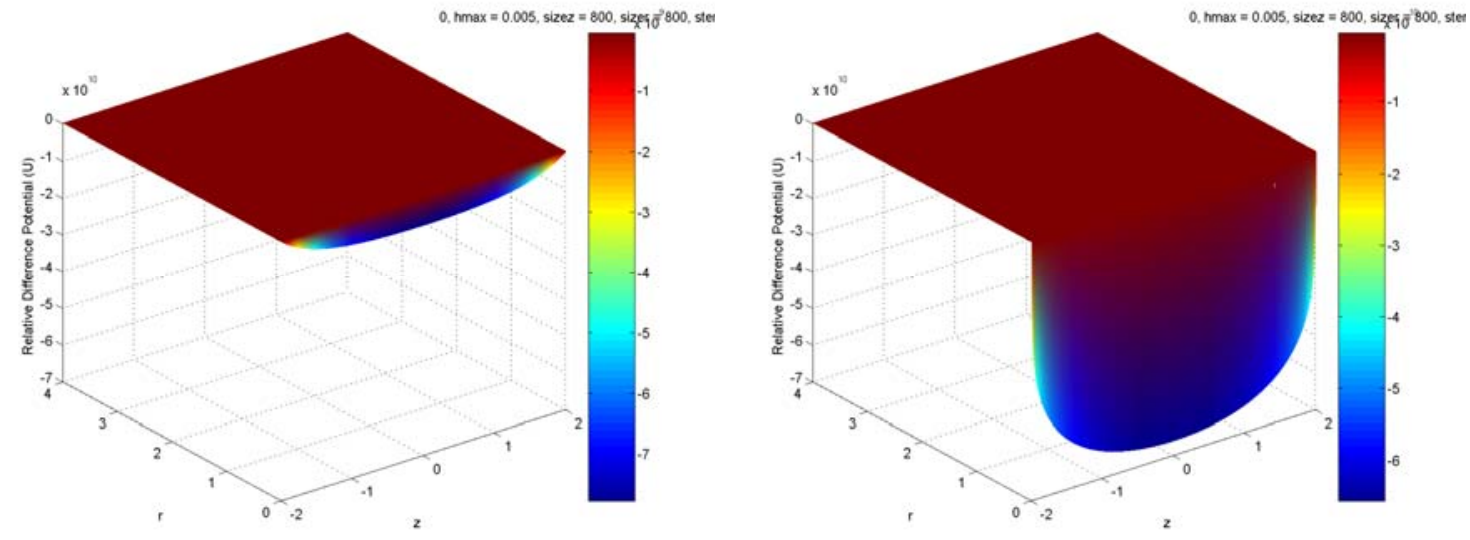

Figure 11: Relative error for the FD (left) and LTP (right) schemes for an 800x800 cell mesh for the bounded case. 
The rate of convergence and the trend-line for the maximum relative error in the periodic case is shown in the left-hand graph and for the non-periodic case in the right-hand graph of Fig. 12. The periodic FD version has a trend-line of $y=1.8972 x+37.7373$ and the non-periodic FD version has a trend-line of $y=1.8799 x+37.4851$. The trend-line is $y=2.0381 x+37.5762$ for the periodic LTP and $\mathrm{y}=2.037 \mathrm{x}+36.3862$ for the non-periodic LTP case. The LTP converges more quickly in both cases, demonstrating the ability of LTP to yield lower errors with complex source distributions.
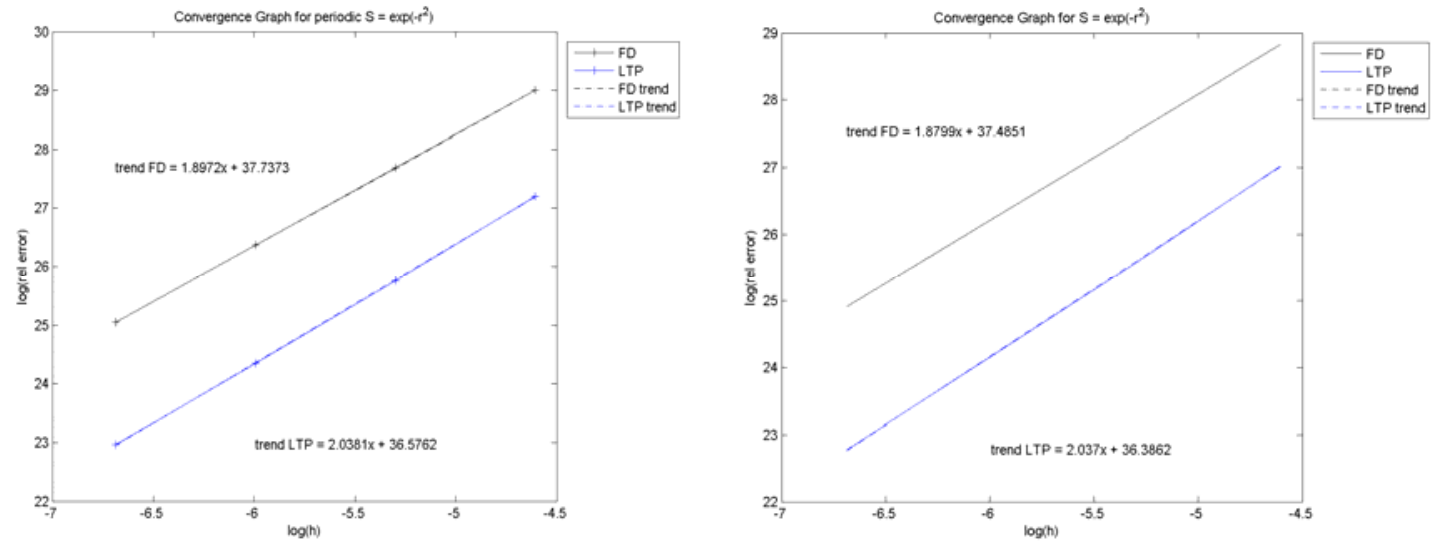

Figure 12: Convergence rate for FD and LTP for periodic (left) and bounded (right) cases.

\section{Extend LTP Algorithms to 3-D}

In Cartesian coordinates extending LTP to three dimensions is straightforward, although massively tedious, and all of the matrix techniques developed previously carry over directly. As above, we start with a general polynomial written in three offset coordinates, shown below, where $\left(x_{p}, y_{p}, z_{p}\right)$ is the primary node. Again, we emphasize that the polynomial is written in this form in order to have a common designation for coefficient and solution order, and a direct correspondence to the ordered terms in the Taylor series of $U$.

$$
\begin{gathered}
v=\left(x-x_{p}\right) / h \quad \eta=\left(y-y_{p}\right) / h \quad \varepsilon=\left(z-z_{p}\right) / h \\
U(v, \eta, \varepsilon)=\sum_{i=0}^{\infty} \sum_{j=0}^{i} \sum_{k=0}^{i-j} A_{i j k} v^{i-j} \eta^{j-k} \varepsilon^{k}
\end{gathered}
$$

Because of the one-to-one correspondence between this polynomial and the Taylor expansion of $U$ about the primary node, it is clear that each coefficient, for example $A_{m n l}$, includes an implied power of the scale factor, $h^{m}$, equal to the coefficient order.

The Poisson PDE in local Cartesian coordinates can be written as shown below along with the source polynomial.

$$
\begin{gathered}
\frac{\partial^{2} U}{\partial v^{2}}+\frac{\partial^{2} U}{\partial \eta^{2}}+\frac{\partial^{2} U}{\partial \varepsilon^{2}}=-4 \pi h^{2} \rho=-S(v, \eta, \varepsilon) \\
S(v, \eta, \varepsilon)=\sum_{i=0}^{\infty} \sum_{j=0}^{i} \sum_{k=0}^{i-j} B_{i j k} v^{i-j} \eta^{j-k} \varepsilon^{k}
\end{gathered}
$$


Substitution of the polynomial expressions for $U$ and $S$ into the PDE yields relationships among the coefficients as in the previous 1-D and 2-D cases. Other then the magnitude and tediousness of the algebra involved, there are no significant differences between the 3D and the lower dimensional cases in Cartesian coordinates. Coefficient reduction, solution weights, electric field calculation, interpolation, non-conformal boundaries, etc. can all be approached using the matrix techniques developed above. All that is required is to define an ordered process for placing the coefficients into the array $\mathbf{A}$ with the corresponding offset terms ordered in the same fashion in a row of the offset matrix $\mathbf{X}$.

In cylindrical coordinates there are significant differences between the 2-D $(r, z)$ and 3-D $(r, \theta, z)$ cases. The scalar Poisson equation in 3-D cylindrical coordinates is shown below.

$$
\frac{\partial^{2} U}{\partial r^{2}}+\frac{1}{r} \frac{\partial U}{\partial r}+\frac{1}{r^{2}} \frac{\partial^{2} U}{\partial \theta^{2}}+\frac{\partial^{2} U}{\partial z^{2}}=-4 \pi \rho
$$

Localization of this PDE presents a few issues. First there is a physical requirement that values must be identical after rotation by $2 \pi$. Preserving this feature at the local level is desirable, even if not a mathematical necessity. Another issue is length computation. In the Cartesian case it is clear that $d^{2}=v^{2}+\eta^{2}+\varepsilon^{2}$ represents the distance from the primary node to any local point. Again, this would be a desirable feature for any set of local variables in cylindrical coordinates.

The local " $r$ " and " $z$ " variables in 3D cylindrical coordinates are the same as for the $2 \mathrm{D}$ case. Substituting just these two variables into the PDE yields:

$$
\frac{\partial^{2} U}{\partial \eta^{2}}+\frac{\delta}{(1+\delta \eta)} \frac{\partial U}{\partial \eta}+\frac{\delta^{2}}{(1+\delta \eta)^{2}} \frac{\partial^{2} U}{\partial \theta^{2}}+\frac{\partial^{2} U}{\partial \varepsilon^{2}}=-S
$$

The most straightforward local variable for the azimuthal direction is the normalized arc difference, defined below. This does not provide the rotational identity and distance features but is easy to implement and does not further complicate the localized PDE.

$$
\begin{gathered}
v=\frac{r_{P}\left(\theta-\theta_{p}\right)}{h} \\
\frac{\partial^{2} U}{\partial \eta^{2}}+\frac{\delta}{(1+\delta \eta)} \frac{\partial U}{\partial \eta}+\frac{1}{(1+\delta \eta)^{2}} \frac{\partial^{2} U}{\partial v^{2}}+\frac{\partial^{2} U}{\partial \varepsilon^{2}}=-S
\end{gathered}
$$

Another possibility that was examined briefly provides some of the rotational and distance features and is shown below. It yields a distance formula that is similar in form to the Cartesian case and has rotational identity, but at $4 \pi$ rotation. However, this choice complicates the localized PDE.

$$
\begin{gathered}
v=\frac{2}{\delta} \sin \left(\frac{\theta-\theta_{p}}{2}\right) \\
d^{2}=\varepsilon^{2}+\eta^{2}+(1+\delta \eta) v^{2}
\end{gathered}
$$

These issues will be examined in more detail in the Phase II effort.

\section{Extend LTP Technique to Electromagnetic Regime}


The LTP algorithm can be extended to the electromagnetic regime in one of two schemes. First, the underlying expansions can be written with a time variable in the expansion, using the resulting spatio-temporal polynomial expression directly in Maxwell's equations. Second, the time-independent spatial LTP algorithm can be used in Maxwell's equations with a finite difference for the temporal part.

In the first scheme, taking one spatial dimension for simplicity, the normalized space and time variables become:

$$
\eta=\left(x-x_{p}\right) / h_{x}, \tau=\left(t-t_{p}\right) / h_{t},
$$

where $x_{\mathrm{p}}$ and $t_{\mathrm{p}}$ are the local position and time references, and $h_{\mathrm{x}}$ and $h_{\mathrm{t}}$ are the local space and time step, respectively.

Using the definitions of $\eta$ and $\tau$, Maxwell's equations can be written in one dimension:

$$
\begin{aligned}
& \partial_{\tau} \vec{H}=-\frac{h_{x}}{\mu_{0} h_{t}} \nabla_{\eta} \times \vec{E} \\
& \partial_{\tau} \vec{E}=\frac{h_{x}}{\varepsilon_{0} h_{t}} \nabla_{\eta} \times \vec{H}-\frac{\vec{J}}{\varepsilon_{0} h_{t}}
\end{aligned}
$$

where $\mu_{0}$ and $\varepsilon_{0}$ are the permeability and permittivity of free space, respectively.

The fields and current density can be written as LTPs:

$$
\begin{aligned}
& \vec{E}(\eta, \tau)=\sum_{i=0}^{M} \sum_{j=0}^{i} \vec{A}_{i, j} \tau^{i-j} \eta^{j} \\
& \vec{H}(\eta, \tau)=\sum_{i=0}^{M} \sum_{j=0}^{i} \vec{B}_{i, j} \tau^{i-j} \eta^{j} \\
& \vec{J}(\eta, \tau)=\sum_{i=0}^{N} \sum_{j=0}^{i} \vec{C}_{i, j} \tau^{i-j} \eta^{j}
\end{aligned}
$$

Combining, we obtain the LTP form of the Maxwell curl eqs.:

$$
\sum_{i=0}^{M} \sum_{j=0}^{i-1} \vec{B}_{i, j}(i-j) \tau^{i-j-1} \eta^{j}=-\frac{h_{x}}{\mu_{0} h_{t}}\left(\begin{array}{c}
0 \\
-\sum_{i=0}^{M} \sum_{j=1}^{i-1} j A_{z, i, j} \tau^{i-j} \eta^{j-1} \\
\sum_{i=0}^{M} \sum_{j=1}^{i-1} j A_{y, i, j} \tau^{i-j} \eta^{j-1}
\end{array}\right)
$$




$$
\sum_{i=0}^{M} \sum_{j=0}^{i-1} \vec{A}_{i, j}(i-j) \tau^{i-j-1} \eta^{j}=\frac{h_{x}}{\mu_{0} h_{t}}\left(\begin{array}{c}
0 \\
\sum_{i=0}^{M} \sum_{j=1}^{M} \sum_{j=1}^{i-1} j B_{z, i, j} \tau^{i-j} \eta^{j-1} \tau^{i-j} \eta^{j-1}
\end{array}\right)-\frac{1}{\varepsilon_{0} h_{t}} \sum_{i=0}^{N} \sum_{j=0}^{i} \vec{C}_{i, j} \tau^{i-j} \eta^{j}
$$

The stability and implementation details of this scheme pose substantial challenges, beyond the scope of the Phase I work.

The second case, in which the left hand side of the LTP form of Maxwell's curl equations is replaced by finite difference operators, follows directly from the above derivation, but poses challenges on the time at which the field components are known.

The challenges of developing the electromagnetic version of the LTP method suggest that while worthwhile, this is a significant project in its own right, and hence should be formulated as a standalone project.

\section{Future Directions: The Phase II Project}

This section outlines the objectives and tasks necessary to convert our Phase I results into usable simulation and design capabilities for photoinjectors.

\subsection{Technical Objectives}

The long-term goal of this project is to develop a flexible, 2D/3D particle-in-cell tool set for complete simulation of photoinjector operation, including the simultaneous time behavior of both the RF accelerating field and the bunch formation/evolution process. Achieving this goal requires implementation of enhanced numerical techniques for representing space charge and fields in cylindrical coordinates and multi-level subgrid and grid patch capabilities. In Phase II, a staged implementation of these capabilities into the OOPIC [4] particle-in-cell code will be done. Starting with 2D and advancing toward full 3D electrostatic, the LTP algorithms developed and tested in Phase I will be implemented to improve solution accuracy and provide a more flexible mesh and boundary architecture in OOPIC.

The Phase II objectives are: 1) implement, validate and apply 2D cylindrical LTP and an improved photo-emission algorithm in OOPIC, and 2) extend LTP to 3D cylindrical coordinates and implement and validate in OOPIC. The target implementation platform, OOPIC, is well tested, widely used and openly available. While, the LTP algorithms developed and tested in Phase I are completely general, implementation into OOPIC will necessarily be code specific. An additional or different target platform can be used if desirable.

\subsection{Proposed Phase II Tasks}

This project will implement high accuracy space charge, subgrid and grid patch algorithms for 2D and 3D cylindrical coordinates in the OOPIC code for improved simulation of photoinjectors. The activities needed to accomplish this are presented below. 


\subsubsection{Task 1: Photoinjector simulations}

This task has two components:

Task-1a: Implement and validate LTP space charge and field algorithms for electrostatic PIC in OOPIC. UCB and CCR will make any necessary modifications and/or extensions to the high-accuracy algorithms for inclusion in the OOPIC code. UCB will be responsible for OOPIC modification and validation testing.

Task-1b: Implement and validate a physics-based, state-of-the-art photoemission algorithm in OOPIC. The basis for the photoemission algorithm will be the model presented in [5].

\subsubsection{Task 2: LTP-based advanced mesh capabilities}

This task will implement and validate multi-level subgrid, grid patch and nonuniform grid algorithms in OOPIC. These algorithms will provide targeted resolution in specific regions of electrostatic simulations. The OOPIC grid enhancements will be tested across a range of relevant subgrid, grid patch and non-uniform grid parameters for accuracy and computational costs.

The advanced grid algorithms will be tested for computational efficiency in electrostatic PIC. The use of multigrids [6,7] and subgrids [8-11] to provide high resolution only where needed is well established in many fields but has not seen widespread use in the PIC community. One of the reasons for this is the complexity of accurately dealing with particles as they enter and exit regions of different resolution. For PIC simulation, where particle pushing is usually the dominant computational cost, this is a nontrivial issue and will be evaluated for the algorithms implemented in OOPIC.

There still remain some basic issues regarding accuracy and particle interpolation for particle trajectories crossing a grid interface. The development of particle algorithms for unstructured grids [7] has lead to research addressing some of the same problems encountered in moving particles between different grid resolutions. In particular, the technique of stopping particles at the interface as they transition between grid regions and switching to fields in the new grid for particle pushing is of interest. So far this algorithm has been used in "ray tracing," which has a distinctly lower computational cost than PIC, and, hence, it must be evaluated for compatibility with electrostatic PIC.

OOPIC is amenable to this technique since it currently detects particle crossings at each cell in order to check for internal boundaries. The grid-subgrid interface can be treated as a special type of internal boundary that triggers appropriate handling of trajectories and space charge across the interface.

\subsubsection{Task 3: Optimize non-uniform mesh capabilities (static)}

Implement and test non-uniform grid capabilities in OOPIC. This task has two primary elements aimed at improving accuracy and computational efficiency: 1) adapting the grid/subgrid/patch to a complex geometry, 2) adapting the 
grid/subgrid/patch to physics. Although the implementation will provide a solid foundation for general adaptive grid techniques, only static grid adaptation will be addressed in this task. Time adaptive grids raise numerous issues for PIC codes and would require too much effort. Providing a validated and useful static adaptive grid capability in Phase II is more important and will act as the basis for further enhancements in this area.

Testing will specifically examine the errors induced in field computation and particle motion when particles cross the interface between regions of different grid resolution. UCB will have primary responsibility for OOPIC modification and testing.

\subsubsection{Task 4: 3D LTP model}

Develop and implement a 3-D LTP method for cylindrical coordinates. In this task, the initial Phase I 3-D investigation will be completed and 3-D LTP solutions methods implemented in OOPIC. A system of local coordinates and the associated field and source polynomials will be developed. Matrix routines for computing and implementing the 3-D cylindrical LTP will be programmed and validated.

\subsubsection{Task 5: Advanced LTP concepts}

Polynomials are often convenient to work with; however, some cases are not well adapted to polynomial representation, requiring a large number of terms for accurate representation. In this task, the possibility of using other sets of basis functions in an LTP-like approach will be examined. Different function sets might prove superior in 3-D, or in other geometries, or with other PDE's (e.g. the reduced Helmholtz PDE), or they might provide more accurate interpolation.

The second element of this task attempts to exploit an intriguing aspect of LTP to improve space charge accuracy. Since LTP views the source as a polynomial, if the polynomial coefficients were known analytically these values could be used directly in the LTP formulas. If particles are defined by a low-order polynomial relative to the particle location, the particle polynomial representation can be analytically shifted to near-by nodes. This concept is easily extended to multiple particles.

\section{Summary and Conclusions}

Photoinjectors in advanced high-energy accelerators reduce beam energy spreads and enhance undulator photon fluxes. Photoinjector design is difficult because of the substantial differences in time and spatial scales. This Phase I program explored an innovative technique, the local Taylor polynomial (LTP) formulation, for improving finite difference analysis of photoinjectors. This included improved weighting techniques, systematic formula for high order interpolation and electric field computation, and improved handling of space charge.

The Phase I results have shown LTP improves accuracy and flexibility for advanced capabilities:

- Powerful, systematic solution weight generation

- Systematic formulation of high order interpolation and electric field computation

- Better handling of space charge gradients 
- Flexibility in application of advanced grid techniques

\section{Bibliography \& References Cited}

[1] I. V. Bazarov, B. M. Dunham, C. Gulliford, Y. Li, X. Liu, C. K. Sinclair, K. Soong, and F. Hannon, "Benchmarking of 3D space charge codes using direct phase space measurements from photoemission high voltage dc gun," Phys. Rev. ST Accel. Beams 11, 100703 (2008).

[2] J. P. Verboncoeur, "Symmetric Spline Weighting for Charge and Current Density in Particle Simulation,” J. Comput. Phys. vol. 174 (2001) pp. 421-427

[3] R. H. Jackson, M. J. McLay and R. P. Joshi, "The Field Marshal Electromagnetic Simulation Environment", final report, Air Force Office of Scientific Research STTR No. FA9550-05-C-0080 (2007)

[4] J. P. Verboncoeur, A. B. Langdon and N. T. Gladd, "An Object-Oriented Electromagnetic PIC Code”, Comput. Phys. Comm. 87, 199-211 (1995).

[5] K. L. Jensen, P. G. O'Shea, D. W. Feldman, and N. A. Moody, "Theoretical model of the intrinsic emittance of a photocathode”, App. Phys. Lett. 89 (2006).

[6] P. Wesseling, An Introduction to Multigrid Methods, R. T. Edwards, Philadelphia, PA USA (2004)

[7] J. Petillo, K. Eppley, D. Panagos, P. Blanchard, E. Nelson, N. Dionne, J. DeFord, B. Held, L. Chernyakova, W. Krueger, S. Humphries, T. Mc- Clure, A. Mondelli, J. Burdette, M. Cattelino, R. True, K. T. Nguyen, and B. Levush, "The MICHELLE Three-Dimensional Electron Gun and Collector Modeling Tool: Theory and Design," IEEE Trans. Plasma Sci., vol. 30, no. 3, pp. 1238-1264, Jun. 2002.

[8] M. W. Chevalier, R. J. Luebbers, "FDTD local grid with material traverse," IEEE Tran. Anten. Prop., 45, 411-421, 1997.

[9] M. Okoniewski, E. Okoniewska and M.A. Stuchly, "Three dimensional subgridding algorithm for FDTD," IEEE Tran. Ant. and Prop., 45, 422-429, 1997.

[10] A. R. Zakharian, M. Brio, and J. V. Moloney, "FDTD Based Second-Order Accurate Local Mesh Refinement Method for Maxwell's Equations in Two Space Dimensions," Comm. Math. Sci. Vol. 2, No. 3, pp. 497-513, 2004.

[11] A. Taflove and S. C. Hagness, Computational Electrodynamics: the Finite Difference Time Domain Method, 3rd ed., Arthech House, Boston; London (2005) 